\title{
SMOOTHING OPERATORS IN MULTI-MARGINAL OPTIMAL TRANSPORT
}

\author{
UGO BINDINI
}

\begin{abstract}
Given $N$ absolutely continuous probabilities $\rho_{1}, \ldots, \rho_{N}$ over $\mathbb{R}^{d}$ which have Sobolev regularity, and given a transport plan $P$ with marginals $\rho_{1}, \ldots, \rho_{N}$, we provide a universal technique to approximate $P$ with Sobolev regular transport plans with the same marginals. Moreover, we prove a sharp control of the energy and some continuity properties of the approximating family.
\end{abstract}

\section{INTRODUCTION}

We consider a multi-marginal Optimal Transport problem on the Euclidean space: given $N$ Borel probability measures $\rho_{1}, \ldots, \rho_{N} \in \mathcal{P}\left(\mathbb{R}^{d}\right)$, and given a cost function $c:\left(\mathbb{R}^{d}\right)^{N} \rightarrow \mathbb{R}$, the goal is to find

$$
\min _{P} \int c\left(x_{1}, \ldots, x_{N}\right) \mathrm{d} P\left(x_{1}, \ldots, x_{N}\right)
$$

under the constraint

$$
P \in \Pi\left(\rho_{1}, \ldots, \rho_{N}\right):=\left\{P \in \mathcal{P}\left(\left(\mathbb{R}^{d}\right)^{N}\right) \mid \pi_{\#}^{j} P=\rho_{j} \forall j=1, \ldots, N\right\} .
$$

Here $\pi^{j}:\left(\mathbb{R}^{d}\right)^{N} \rightarrow \mathbb{R}^{d}$ denotes the projection onto the $j$-th coordinate, i.e., $\pi^{j}\left(x_{1}, \ldots, x_{N}\right)=$ $x_{j}$.

When $N=2$, the classical Kantorovich formulation of the Optimal Transport problem is recovered; however, many characteristics of the multi-marginal problem are different from the classical one. For a good survey on both cases see for instance [1, 10.

In this work we want to investigate the properties of the space $\Pi\left(\rho_{1}, \ldots, \rho_{N}\right)$ when the measures $\rho_{1}, \ldots, \rho_{N}$ share some regularity - in particular, we are interested in the case when the marginals have a Sobolev-type regularity, as clarified in the following

Definition 1. If $p \geq 1$, we say that a probability measure $\mu \in \mathcal{P}\left(\mathbb{R}^{m}\right)$ is $W^{1, p_{\text {- }}}$ regular if $\mu$ is absolutely continuous with respect to the Lebesgue measure $\mathcal{L}^{m}$, and

$$
\left(\frac{\mathrm{d} \mu}{\mathrm{d} \mathcal{L}^{m}}\right)^{\frac{1}{p}} \in W^{1, p}\left(\mathbb{R}^{m}\right) .
$$

In other words, $\mu$ is $W^{1, p}$-regular if there exists $f \in W^{1, p}\left(\mathbb{R}^{m}\right), f \geq 0$, such that

$$
\frac{\mathrm{d} \mu}{\mathrm{d} \mathcal{L}^{m}}=f^{p} .
$$

We will denote by $\mathcal{P}^{1, p}\left(\mathbb{R}^{m}\right)$ the space of $W^{1, p}$-regular probability measures. This definition arises naturally in the setting of Density Functional Theory as a generalization of the one given by Lieb in $[9$ for $p=2$. In what follows, when we say that a measure is regular we will mean that it is $W^{1, p}$-regular for some fixed $p$. After giving some basic notation and results in Section 2, we study in Section 3

Date: March 26, 2019 
the properties of regular measures, stressing in particular the relation between a measure and its marginals.

Even when the marginals $\rho_{1}, \ldots, \rho_{N}$ are regular, the optimal plan in (1.1) may be singular; it is well known, for instance, that in the case $N=2$, under suitable hypotheses, the optimal plan is concentrated on a graph. On the other hand, for many applications, and in particular when dealing with $\Gamma$-convergence, it may be useful to construct regular transport plans which are "close" to a given optimal one (see for instance [4, 7, 8]). With this in mind, in Sections 5] 8, we address the following

Problem: Given $\rho_{1}, \ldots, \rho_{N} \in \mathcal{P}^{1, p}\left(\mathbb{R}^{d}\right)$, and given $\mu \in \Pi\left(\rho_{1}, \ldots, \rho_{N}\right)$, find $a$ family $\left(\mu^{\varepsilon}\right)_{\varepsilon>0}$ such that:

(i) $\mu^{\varepsilon} \in \Pi\left(\rho_{1}, \ldots, \rho_{N}\right)$;

(ii) $\mu^{\varepsilon} \in \mathcal{P}^{1, p}\left(\left(\mathbb{R}^{d}\right)^{N}\right)$;

(iii) $\mu^{\varepsilon} \rightarrow \mu$ as $\varepsilon \rightarrow 0$ (for a suitable notion of convergence).

In other words, we search for $W^{1, p}$-regular multi-marginal transport plans with marginals $\rho_{1}, \ldots, \rho_{N}$ which approximate a (non regular) transport plan $\mu$. Since in general $\mu$ could be no more regular than a measure, the natural topology for (iii) is the tight convergence of probability measures, i.e., weak convergence in duality with $C_{b}\left(\left(\mathbb{R}^{d}\right)^{N}\right)$ (continuous and bounded functions).

Notice that, if $\mu$ is optimal in (1.1), and the cost $c$ is upper semi-continuous and bounded from above, combining (iii) and the Portmanteau's Theorem we get

$$
\lim _{\varepsilon \rightarrow 0} \int c(X) \mathrm{d} \mu^{\varepsilon}(X)=\int c(X) \mathrm{d} \mu(X)
$$

whence we may say that $\mu^{\varepsilon}$ is "almost" optimal for small $\varepsilon$.

This problem has already been treated in C. Cotar, G. Friesecke and C. Klüppelberg in [6, 7] and solved with a different construction for $p=2$. Our technique was introduced in collaboration with L. De Pascale in [3] and later used in [4] for studying the semiclassical limit in Density Functional Theory. Recently, our construction was extended to mixed states by M. Lewin in [8]. In the present work we give a systematic presentation of the results for general $p \geq 1$, and we are also able to obtain sharp energy estimates (Theorem 6.3) and Theorem 6.4) and a strong $W^{1, p_{-}}$ continuity property (Theorem 5.1). The latter, in particular, turns out to be a very useful tool in order to study the properties of the mapping between a transport plan and its marginals. We will use it, in a forthcoming work in preparation with L. De Pascale, to show that the map which sends a symmetric wave-function to its marginal is open, partially answering to a conjecture posed by Lieb in [9, Question $2]$.

Finally, we want to point out that the definition of the smoothing operator (Section 5), which we give in the case of Sobolev spaces due to physical interest, works in the same way for other classes of absolutely continuous measures, e.g., measures with $C^{k, \alpha}$ density, with analogous regularity and continuity results.

\section{Notation AND PRELIMINARY RESUlts}

We will denote by $\mathbb{R}^{+}$the open interval $(0,+\infty)$. We recall the following elementary inequalities, valid for any $a, b \geq 0$ :

$$
\begin{aligned}
& \left|a^{p}-b^{p}\right| \leq|a-b||a+b|^{p-1} \quad 1 \leq p<\infty \\
& \left|a^{\gamma}-b^{\gamma}\right| \leq|a-b|^{\gamma} \quad 0<\gamma \leq 1 \text {. }
\end{aligned}
$$


Given $\mu \in \mathcal{P}\left(\left(\mathbb{R}^{d}\right)^{N}\right)$, we denote its marginals by $\left.\mu\right|_{j}:=\pi_{\#}^{j} \mu$, for $j=1, \ldots, N$. If $f:\left(\mathbb{R}^{d}\right)^{N} \rightarrow \mathbb{R}$, and $1 \leq j \leq N$, we denote by

$$
\int f(X) \mathrm{d} \hat{X}_{j}:=\int f\left(x_{1}, \ldots, x_{N}\right) \mathrm{d} x_{1} \cdots \mathrm{d} \hat{x}_{j} \cdots \mathrm{d} x_{N}
$$

the integral of $f$ with respect to all the variables except $x_{j}$. This is a function of the variable $x_{j}$.

When $f \in W^{1, p}\left(\mathbb{R}^{m}\right)$, we will denote by

$$
|\nabla f|:=\left(\sum_{j=1}^{m}\left|\partial_{x_{j}} f\right|^{p}\right)^{\frac{1}{p}},
$$

i.e., when computing the norm of a gradient we take on $\mathbb{R}^{m}$ the $p$-th norm.

We say that a sequence of probability measures $\left\{\mu_{k}\right\} \subseteq \mathcal{P}\left(\mathbb{R}^{m}\right)$ weakly converges to $\mu \in \mathcal{P}\left(\mathbb{R}^{m}\right)$, denoted $\mu_{k} \rightarrow \mu$, if for every $\phi \in C_{b}\left(\mathbb{R}^{m}\right)$

$$
\lim _{k \rightarrow \infty} \int \phi \mathrm{d} \mu_{k}=\int \phi \mathrm{d} \mu .
$$

A family of measures $\mathcal{M} \subseteq \mathcal{P}\left(\mathbb{R}^{m}\right)$ is said to be tight if for every $\delta>0$ there exists $K \subseteq \mathbb{R}^{m}$ compact such that $\mu(K) \geq 1-\delta$ for every $\mu \in \mathcal{M}$.

Finally we recall the following classical results.

Theorem 2.1 (Prokhorov's theorem). A family $\mathcal{M} \subseteq \mathcal{P}\left(\mathbb{R}^{m}\right)$ is tight if and only if for every sequence $\left\{\mu_{k}\right\} \subseteq \mathcal{M}$ there exists a subsequence $\left\{\mu_{n_{k}}\right\}$ and $\mu \in \mathcal{P}\left(\mathbb{R}^{m}\right)$ with $\mu_{n_{k}} \rightarrow \mu$.

Theorem 2.2 (Generalized Lebesgue's dominated convergence theorem). Let $\left\{f_{n}\right\}_{n \in \mathbb{N}}$ and $\left\{g_{n}\right\}_{n \in \mathbb{N}}$ be Lebesgue measurable functions, with $g_{n} \geq 0$. Suppose that:

(i) $\left|f_{n}(x)\right| \leq g_{n}(x)$ for all $n \in \mathbb{N}$, for almost every $x$;

(ii) $\left\{f_{n}\right\}$ converges pointwise almost everywhere to $f$ and $\left\{g_{n}\right\}$ converges pointwise almost everywhere to $g$;

(iii)

$$
\lim _{n \rightarrow \infty} \int g_{n}=\int g
$$

Then $f$ is Lebesgue integrable on $E$ and

$$
\lim _{n \rightarrow \infty} \int f_{n}=\int f
$$

2.1. Roots and powers of non-negative Sobolev functions. The following Propositions will be useful later in order to have an expression for the weak derivatives of $p$-th powers and $p$-th roots of non-negative Sobolev functions.

Proposition 2.3. Let $p>1$. If $u \in W^{1, p}\left(\mathbb{R}^{m}\right), u \geq 0$, then $u^{p} \in W^{1,1}\left(\mathbb{R}^{m}\right)$, and $\nabla u^{p}=p u^{p-1} \nabla u$.

Viceversa, let $u \in W^{1,1}\left(\mathbb{R}^{m}\right), u \geq 0$, such that

$$
\int u^{1-p}|\nabla u|^{p}<\infty
$$

Then $u^{\frac{1}{p}} \in W^{1, p}\left(\mathbb{R}^{m}\right)$, and $\nabla u^{\frac{1}{p}}=\frac{1}{p} u^{\frac{1-p}{p}} \nabla u$.

Proof. If $u \in W^{1, p}\left(\mathbb{R}^{m}\right)$ clearly $u^{p} \in L^{1}\left(\mathbb{R}^{m}\right)$, and viceversa if $u \in W^{1,1}\left(\mathbb{R}^{m}\right)$ then $u^{\frac{1}{p}} \in L^{p}\left(\mathbb{R}^{m}\right)$. Let $u_{n} \in C^{\infty}\left(\mathbb{R}^{m}\right) \cap W^{1, p}\left(\mathbb{R}^{m}\right)$ such that $u_{n} \rightarrow u$ in $W^{1, p}\left(\mathbb{R}^{m}\right)$. 
Then by the Hölder inequality with exponents $p$ and $\frac{p}{p-1}$

$$
\begin{aligned}
\int\left|u_{n}^{p-1} \nabla u_{n}-u^{p-1} \nabla u\right| & \leq \int u_{n}^{p-1}\left|\nabla u_{n}-\nabla u\right|+\int|\nabla u|\left|u_{n}^{p-1}-u^{p-1}\right| \\
& =\left\|u_{n}\right\|_{p}^{p-1}\left\|\nabla u-\nabla u_{n}\right\|_{p}+\|\nabla u\|_{p}\left\|\left|u_{n}^{p-1}-u^{p-1}\right|^{\frac{1}{p-1}}\right\|_{p}^{p-1} .
\end{aligned}
$$

If $p \geq 2$ we use (2.1) and the Hölder inequality to get

$$
\left\|\left|u_{n}^{p-1}-u^{p-1}\right|^{\frac{1}{p-1}}\right\|_{p}^{p-1} \leq\left\|u_{n}-u\right\|_{p}\left\|u_{n}+u\right\|_{p}^{p-2}
$$

if $1<p<2$, let $\gamma=p-1 \in(0,1)$ and use (2.2) to get

$$
\left\|\left|u_{n}^{p-1}-u^{p-1}\right|^{\frac{1}{p-1}}\right\|_{p}^{p-1} \leq\left\|u_{n}-u\right\|_{p}^{p-1} .
$$

This completes the proof of the first part. Suppose on the contrary that $u \in$ $W^{1,1}\left(\mathbb{R}^{m}\right), u \geq 0$, and that the condition (2.4) holds. Fix $\phi \in C_{c}^{\infty}\left(\mathbb{R}^{m}\right)$ and $\varepsilon>0$. We want to prove that

$$
\int(u+\varepsilon)^{\frac{1}{p}} \nabla \phi=-\frac{1}{p} \int \phi(u+\varepsilon)^{\frac{1-p}{p}} \nabla u .
$$

To this end, let $u_{n} \rightarrow u$ in $W^{1,1}\left(\mathbb{R}^{m}\right)$, where $u_{n} \in C^{\infty}, u_{n} \geq 0$; up to a subsequence we may suppose also $u_{n} \rightarrow u$ and $\nabla u_{n} \rightarrow \nabla u$ pointwise almost everywhere. Putting $u_{n}$ in place of $u$ in (2.5) we have pointwise convergence of both the integrands, and we conclude via Theorem 2.2 using the dominations

$$
\left|\phi\left(u_{n}+\varepsilon\right)^{\frac{1-p}{p}} \nabla u_{n}\right| \leq \varepsilon^{\frac{1-p}{p}}|\phi|\left|\nabla u_{n}\right|, \quad\left|\phi(u+\varepsilon)^{\frac{1-p}{p}} \nabla u\right| \leq \varepsilon^{\frac{1-p}{p}}|\phi||\nabla u| .
$$

Finally, letting $\varepsilon \rightarrow 0$ in (2.5), we have once again pointwise convergence of the integrands, and we conclude by the classical Lebesgue's dominated covergence Theorem thanks to the hypothesis and the domination

$$
\left|\phi(u+\varepsilon)^{\frac{1-p}{p}} \nabla u\right|^{p} \leq|\phi|^{p} u^{1-p}|\nabla u|^{p} .
$$

Note that the condition (2.4) in Proposition 2.3 is necessary, as the following example shows.

Example 1. In dimension $m=1$, fix $p>1$ and consider the $W^{1,1}$ function

$$
f(x)= \begin{cases}\sin (x)^{p-1} & 0 \leq x \leq \pi \\ 0 & \text { otherwise }\end{cases}
$$

whose weak derivative is $f^{\prime}(x)=\chi_{[0, \pi]} \sin (x)^{p-2} \cos (x)$. The point is that $f^{\frac{1}{p}}$ does not belong to $W^{1, p}(\mathbb{R})$, since the weak derivative of $f^{\frac{1}{p}}$ should be $g_{p}(x)=$ $\frac{p-1}{p} \chi_{[0, \pi]} \sin (x)^{-\frac{1}{p}} \cos (x)$, but

$$
\int_{0}^{\pi}\left|g_{p}(x)^{p}\right| \mathrm{d} x=\frac{(p-1)^{p}}{p^{p}} \int_{0}^{\pi} \frac{|\cos (x)|^{p}}{\sin (x)} \mathrm{d} x
$$

diverges at both 0 and $\pi$

Proposition 2.4. If $u_{n} \rightarrow u$ in $W^{1, p}\left(\mathbb{R}^{m}\right), u_{n}, u \geq 0$, then $u_{n}^{p} \rightarrow u^{p}$ in $W^{1,1}\left(\mathbb{R}^{m}\right)$.

Viceversa, let $u_{n} \rightarrow u$ in $W^{1,1}\left(\mathbb{R}^{d}\right), u_{n}, u \geq 0$. Let $h_{n}, h \in L^{1}\left(\mathbb{R}^{m}\right)$ such that $u_{n}^{1-p}\left|\nabla u_{n}\right|^{p} \leq h_{n}, u^{1-p}|\nabla u|^{p} \leq h$, and

$$
\lim _{n \rightarrow \infty} \int h_{n}=\int h .
$$

Suppose also that for every subsequence $\left\{h_{n_{k}}\right\}$ there exists a further subsequence converging to $h$ pointwise a.e. Then $u_{n}^{\frac{1}{p}} \rightarrow u^{\frac{1}{p}}$ in $W^{1, p}\left(\mathbb{R}^{m}\right)$. 
Proof. If $p=1$ there is nothing to prove, so assume $p>1$, and take $u_{n} \rightarrow u$ in $W^{1, p}\left(\mathbb{R}^{m}\right)$. Using (2.1) and the Hölder inequality with exponents $p$ and $\frac{p}{p-1}$,

$$
\int\left|u_{n}^{p}-u^{p}\right| \leq\left\|u_{n}-u\right\|_{p}\left\|u_{n}+u\right\|_{p}^{p-1} .
$$

Since $u_{n} \rightarrow u$ in $W^{1, p}\left(\mathbb{R}^{m}\right)$ and hence in particular $u_{n}$ is bounded in $L^{p}\left(\mathbb{R}^{m}\right)$, we get that $u_{n}^{p} \rightarrow u^{p}$ (strongly) in $L^{1}\left(\mathbb{R}^{m}\right)$.

Moreover, $\nabla u_{n}^{p}=p u_{n}^{p-1} \nabla u_{n}$ and $\nabla u^{p}=p u^{p-1} \nabla u$ by Proposition 2.3, hence by the Hölder inequality

$$
\begin{aligned}
\int\left|\nabla u_{n}^{p}-\nabla u^{p}\right| & \leq p \int u_{n}^{p-1}\left|\nabla u_{n}-\nabla u\right|+p \int|\nabla u|\left|u_{n}^{p-1}-u^{p-1}\right| \\
& \leq p\left\|u_{n}\right\|_{p}^{p-1}\left\|\nabla u_{n}-\nabla u\right\|_{p}+p \int|\nabla u|\left|u_{n}^{p-1}-u^{p-1}\right|,
\end{aligned}
$$

which converges to zero as in the proof of Proposition 2.3.

To prove the converse, suppose by contradiction that there is a subsequence (denoted again $u_{n}$ ) such that

$$
\left\|u_{n}^{\frac{1}{p}}, u^{\frac{1}{p}}\right\|_{W^{1, p}} \geq \delta>0 .
$$

By hypothesis, up to a further subsequence we may assume that $u_{n_{k}} \rightarrow u$, $\nabla u_{n_{k}} \rightarrow \nabla u$ and $h_{n_{k}} \rightarrow h$ pointwise almost everywhere. Then we have by (2.2), with $\gamma=\frac{1}{p}$,

$$
\int\left|u_{n_{k}}^{\frac{1}{p}}-u^{\frac{1}{p}}\right|^{p} \leq \int\left|u_{n_{k}}-u\right|=\left\|u_{n_{k}}-u\right\|_{1}
$$

and

$$
\left\|\nabla u_{n_{k}}^{\frac{1}{p}}-\nabla u^{\frac{1}{p}}\right\|_{p}=\frac{1}{p^{p}} \int\left|u_{n_{k}^{\frac{1-p}{p}}} \nabla u_{n_{k}}-u^{\frac{1-p}{p}} \nabla u\right|^{p} .
$$

Here the integrand converges to zero pointwise, and using the domination

$$
\left|u_{n_{k}}^{\frac{1-p}{p}} \nabla u_{n_{k}}-u^{\frac{1-p}{p}} \nabla u\right|^{p} \leq 2^{p-1}\left(u_{n_{k}}^{1-p}\left|\nabla u_{n_{k}}\right|^{p}+u^{1-p}|\nabla u|^{p}\right) \leq 2^{p-1}\left(h_{n_{k}}+h\right)
$$

and the condition (2.6) we conclude thanks to Theorem 2.2 that $u_{n_{k}}^{\frac{1}{p}} \rightarrow u^{\frac{1}{p}}$ in $W^{1, p}\left(\mathbb{R}^{m}\right)$, contradicting (2.7).

\section{Regular measures}

In this Section we study the space $\mathcal{P}^{1, p}\left(\mathbb{R}^{m}\right)$ of $W^{1, p}$-regular measures. By Proposition 2.3, it is immediate to see that

$$
\mu \in \mathcal{P}^{1, p}\left(\mathbb{R}^{m}\right) \Longrightarrow \mu \in \mathcal{P}^{1,1}\left(\mathbb{R}^{m}\right),
$$

but the converse is not true in general if $p>1$ (see Example 1). Thus, when $p>1$ we have a strict inclusion $\mathcal{P}^{1, p}\left(\mathbb{R}^{m}\right) \subsetneq \mathcal{P}^{1,1}\left(\mathbb{R}^{m}\right)$. .

The set $\mathcal{P}^{1, p}\left(\mathbb{R}^{m}\right)$ has a natural structure of metric space if endowed with the distance

$$
d^{1, p}(\mu, \nu)=\left\|\left(\frac{\mathrm{d} \mu}{\mathrm{d} \mathcal{L}^{m}}\right)^{\frac{1}{p}}-\left(\frac{\mathrm{d} \nu}{\mathrm{d} \mathcal{L}^{m}}\right)^{\frac{1}{p}}\right\|_{W^{1, p}},
$$

which can be seen as a refined version of the Hellinger distance between two absolutely continuous probability measures, where the $L^{p}$ norm of the $p$-th roots is replaced by the $W^{1, p}$ norm. 
We aim to study the space $\left(\mathcal{P}^{1, p}\left(\left(\mathbb{R}^{d}\right)^{N}\right), d^{1, p}\right)$ in relation with the map which sends a $W^{1, p}$-regular probability onto its marginals, namely

$$
\begin{aligned}
\pi: \mathcal{P}^{1, p}\left(\left(\mathbb{R}^{d}\right)^{N}\right) & \longrightarrow \mathcal{P}\left(\mathbb{R}^{d}\right)^{N} \\
\mu & \left.\longmapsto\left(\mu \iota_{1}, \ldots, \mu\right\rfloor_{N}\right) .
\end{aligned}
$$

In particular we want to prove the two following facts:

- if $\mu$ is $W^{1, p}$-regular, then $\mu L_{j}$ is $W^{1, p}$-regular for every $j=1, \ldots, N$;

- the map $\pi: \mathcal{P}^{1, p}\left(\left(\mathbb{R}^{d}\right)^{N}\right) \longrightarrow \mathcal{P}^{1, p}\left(\mathbb{R}^{d}\right)^{N}$ is continuous with respect to the distance $d^{1, p}$ and the relative product topology on the codomain.

These properties will be proved in Theorem 3.2 and Theorem 3.4 respectively. We remark that the latter was alredy proved by Brezis in [9, Appendix] in the case $p=2$. We start by introducing some technical results about the projection map. In what follows, if $\mu$ is $W^{1, p}$-regular, with a slight abuse of notation we will denote by $\mu(X)$ its density, whose $p$-th root belongs to $W^{1, p}\left(\left(\mathbb{R}^{d}\right)^{N}\right)$. For $j=1, \ldots, N$ let

$$
\mu \iota_{j}\left(x_{j}\right)=\int \mu(X) \mathrm{d} \hat{X}_{j}, \quad \nabla \mu \iota_{j}\left(x_{j}\right)=\int \nabla_{x_{j}} \mu(X) \mathrm{d} \hat{X}_{j}
$$

where $\nabla_{x_{j}} \mu$ is defined according to Proposition 2.3. It is easy to prove, approximating $\mu$ with smooth functions in $W^{1,1}\left(\left(\mathbb{R}^{d}\right)^{N}\right)$, that $\nabla \mu l_{j}$ is the distributional gradient of $\left.\mu\right|_{j}$, hence $\mu \iota_{j} \in W^{1,1}\left(\mathbb{R}^{d}\right)$.

Remark 1 . Notice that $\mu \iota_{j}$ coincides with the (density of the) push-forward measure under the projection $\pi^{j}:\left(\mathbb{R}^{d}\right)^{N} \rightarrow \mathbb{R}^{d}$ on the $j$-th factor, which makes the notation is consistent.

By Proposition 2.3, in order to prove that the marginals of a $W^{1, p}$-regular measure are $W^{1, p}$-regular, it suffices to show that

$$
\int \mu L_{j}(x)^{1-p}\left|\nabla \mu L_{j}(x)\right|^{p} \mathrm{~d} x
$$

is finite.

Lemma 3.1. Let $\mu \in \mathcal{P}^{1, p}\left(\left(\mathbb{R}^{d}\right)^{N}\right)$. Then, for every $j=1, \ldots, N$,

$$
\mu \iota_{j}\left(x_{j}\right)^{1-p}\left|\nabla \mu \iota_{j}\left(x_{j}\right)\right|^{p} \leq p^{p} \int\left|\nabla_{x_{j}} \mu^{\frac{1}{p}}(X)\right|^{p} \mathrm{~d} \hat{X}_{j} .
$$

Proof. Recalling Proposition 2.3 and using the Hölder inequality with exponents $\frac{p}{p-1}$ and $p$, we get

$$
\begin{aligned}
\left|\nabla \mu \downarrow_{j}\left(x_{j}\right)\right| & \leq p \int \mu(X)^{\frac{p-1}{p}}\left|\nabla_{x_{j}} \mu^{\frac{1}{p}}(X)\right| \mathrm{d} \hat{X}_{j} \\
& \leq p\left(\int \mu(X) \mathrm{d} \hat{X}_{j}\right)^{\frac{p-1}{p}}\left(\int\left|\nabla_{x_{j}} \mu^{\frac{1}{p}}(X)\right|^{p} \mathrm{~d} \hat{X}_{j}\right)^{\frac{1}{p}} \\
& =\left.p \mu\right|_{j}\left(x_{j}\right)^{\frac{p-1}{p}}\left(\int\left|\nabla_{x_{j}} \mu^{\frac{1}{p}}(X)\right|^{p} \mathrm{~d} \hat{X}_{j}\right)^{\frac{1}{p}},
\end{aligned}
$$

which implies the thesis.

As a corollary we obtain

Theorem 3.2. Let $\mu \in \mathcal{P}^{1, p}\left(\left(\mathbb{R}^{d}\right)^{N}\right)$. Then its marginals belong to $\mathcal{P}^{1, p}\left(\mathbb{R}^{d}\right)$, and

$$
\nabla\left(\left.\mu\right|_{j}\right)^{\frac{1}{p}}=\left.\frac{1}{p}\left(\left.\mu\right|_{j}\right)^{\frac{1-p}{p}} \nabla \mu\right|_{j} .
$$

Proof. Apply the result of Proposition 2.3 to $\mu \iota_{j} \in W^{1,1}\left(\mathbb{R}^{d}\right)$, using the domination given by Lemma 3.1. 
Finally we want to prove that the map $\pi$ defined in (3.1) is continuous.

Lemma 3.3. Let $\mu^{n} \rightarrow \mu$ in $\mathcal{P}^{1, p}\left(\left(\mathbb{R}^{d}\right)^{N}\right)$. Then $\left.\left.\mu^{n}\right|_{j} \rightarrow \mu\right|_{j}$ in $L^{1}\left(\mathbb{R}^{d}\right)$ and $\nabla \mu^{n} \iota_{j} \rightarrow \nabla \mu \iota_{j}$ in $L^{1}\left(\mathbb{R}^{d}\right)^{d}$.

Proof. Using (2.1) and the Hölder inequality,

$$
\begin{aligned}
\int\left|\mu^{n} L_{j}\left(x_{j}\right)-\mu L_{j}\left(x_{j}\right)\right| \mathrm{d} x_{j} & =\int\left|\int \mu(X)-\mu^{n}(X) \mathrm{d} \hat{X}_{j}\right| \mathrm{d} x_{j} \\
& \leq \int\left|\mu^{n}(X)-\mu(X)\right| \mathrm{d} X
\end{aligned}
$$

and

$$
\begin{aligned}
\int\left|\nabla \mu^{n} L_{j}\left(x_{j}\right)-\nabla \mu L_{j}\left(x_{j}\right)\right| \mathrm{d} x_{j} & =\int\left|\int \nabla_{x_{j}} \mu^{n}(X)-\nabla_{x_{j}} \mu(X) \mathrm{d} \hat{X}_{j}\right| \mathrm{d} x_{j} \\
& \leq \int\left|\nabla_{x_{j}} \mu^{n}(X)-\nabla_{x_{j}} \mu(X)\right| \mathrm{d} X .
\end{aligned}
$$

We conclude thanks to Proposition 2.3.

Theorem 3.4. The map $\pi$ is continuous from $\mathcal{P}^{1, p}\left(\left(\mathbb{R}^{d}\right)^{N}\right)$ to $\mathcal{P}^{1, p}\left(\mathbb{R}^{d}\right)^{N}$ with the product topology.

Proof. Let $\mu^{n} \rightarrow \mu$ in $\mathcal{P}^{1, p}\left(\left(\mathbb{R}^{d}\right)^{N}\right)$, and fix $j \in\{1, \ldots, N\}$. In order to prove that $\mu^{n} L_{j} \rightarrow \mu L_{j}$ in $\mathcal{P}^{1, p}\left(\mathbb{R}^{d}\right)$ we want to apply Proposition 2.4 with

$$
h_{n}\left(x_{j}\right)=p^{p} \int\left|\nabla_{x_{j}}\left(\mu^{n}\right)^{\frac{1}{p}}(X)\right|^{p} \mathrm{~d} \hat{X}_{j}, \quad h\left(x_{j}\right)=p^{p} \int\left|\nabla_{x_{j}} \mu^{\frac{1}{p}}(X)\right|^{p} \mathrm{~d} \hat{X}_{j} .
$$

By Lemma 3.1 we have $\left(\mu^{n} L_{j}\right)^{1-p}\left|\nabla\left(\mu^{n} L_{j}\right)\right|^{p} \leq h_{n}$ and $\left(\mu l_{j}\right)^{1-p}\left|\nabla \mu l_{j}\right|^{p} \leq h$. Condition (2.6) is ensured by

$$
\begin{aligned}
\lim _{n \rightarrow \infty} \int h_{n}\left(x_{j}\right) \mathrm{d} x_{j} & =p^{p} \lim _{n \rightarrow \infty} \int\left|\nabla_{x_{j}}\left(\mu^{n}\right)^{\frac{1}{p}}(X)\right|^{p} \mathrm{~d} X \\
& =p^{p} \lim _{n \rightarrow \infty}\left\|\nabla_{x_{j}}\left(\mu^{n}\right)^{\frac{1}{p}}\right\|_{p}^{p} \\
& =p^{p}\left\|\nabla_{x_{j}} \mu^{\frac{1}{p}}\right\|_{p}^{p} \\
& =\int h\left(x_{j}\right) \mathrm{d} x_{j} .
\end{aligned}
$$

We now follow a construction similar to the one of the Riesz-Fischer theorem, and already used for the analogous result by Brezis in [9, Appendix]. Recall that, by Proposition 2.4. $\mu^{n} \rightarrow \mu$ in $W^{1,1}\left(\left(\mathbb{R}^{d}\right)^{N}\right)$. For every subsequence (denoted again $\left.h_{n}\right)$, extract a further subsequence $\left(h_{n_{k}}\right)_{k}$ such that:

(i) $\nabla\left(\mu^{n_{k}}\right)^{\frac{1}{p}} \rightarrow \nabla \mu^{\frac{1}{p}}$ pointwise a.e.;

(ii) $\left\|\nabla\left(\mu^{n_{k}}\right)^{\frac{1}{p}}-\nabla \mu^{\frac{1}{p}}\right\|_{L^{p}}^{p} \leq 2^{-k}$.

Let

$$
F(X)=\left|\nabla \mu^{\frac{1}{p}}(X)\right|^{p}+\sum_{k=1}^{\infty}\left|\nabla\left(\mu^{n_{k}}\right)^{\frac{1}{p}}(X)-\nabla \mu^{\frac{1}{p}}(X)\right|^{p} .
$$

Since $F \in L^{1}\left(\left(\mathbb{R}^{d}\right)^{N}\right)$ and clearly

$$
\left|\nabla\left(\mu^{n_{k}}\right)^{\frac{1}{p}}(X)\right|^{p} \leq 2^{p-1} F(X), \quad\left|\nabla \mu^{\frac{1}{p}}(X)\right|^{p} \leq F(X)
$$

we have that $h_{n_{k}} \rightarrow h$ pointwise a.e. by dominated convergence. Finally $\mu^{n} \iota_{j} \rightarrow \mu \iota_{j}$ in $W^{1,1}\left(\mathbb{R}^{d}\right)$ by Lemma 3.3, and we may conclude by Proposition 2.4. 


\section{ENERGy OF REgULAR MEASURES}

If $\mu \in \mathcal{P}^{1, p}\left(\mathbb{R}^{m}\right)$, it will be useful to deal with the Sobolev norm of $\mu^{\frac{1}{p}}$. However, since $\mu$ is a probability,

$$
\left\|\mu^{\frac{1}{p}}\right\|_{W^{1, p}}^{p}=\int \mu(x) \mathrm{d} x+\int\left|\nabla \mu^{\frac{1}{p}}(x)\right|^{p} \mathrm{~d} x=1+\int\left|\nabla \mu^{\frac{1}{p}}(x)\right|^{p} \mathrm{~d} x,
$$

so all the information is contained in the second summand. Therefore we give the following

Definition 2. If $\mu \in \mathcal{P}^{1, p}$, the $W^{1, p}$-energy of $\mu$ is defined as

$$
\mathcal{E}^{1, p}(\mu)=\int\left|\nabla \mu^{\frac{1}{p}}(x)\right|^{p} \mathrm{~d} x .
$$

In the special case $p=2$, this quantity may be seen as the kinetic energy $\int|\nabla \psi|^{2}$ of a system described by a wave-function $\psi \in W^{1,2}\left(\mathbb{R}^{m}\right)$, which justifies the name. It is well-known (see for instance 9]) that the kinetic energy of a wave-function is bounded from below by (a constant times) the kinetic energy of its marginals. This is also true in our setting, as stated in the following

Lemma 4.1. Let $\mu \in \mathcal{P}^{1, p}\left(\left(\mathbb{R}^{d}\right)^{N}\right)$. Then

$$
\mathcal{E}^{1, p}(\mu) \geq \sum_{j=1}^{N} \mathcal{E}^{1, p}\left(\mu \iota_{j}\right)
$$

Moreover, if $\rho_{1}, \ldots, \rho_{N} \in \mathcal{P}^{1, p}\left(\mathbb{R}^{d}\right)$,

$$
\inf \left\{\mathcal{E}^{1, p}(\mu) \mid \mu \in \mathcal{P}^{1, p}\left(\mathbb{R}^{m}\right) \cap \Pi\left(\rho_{1}, \ldots, \rho_{N}\right)\right\}=\sum_{j=1}^{N} \mathcal{E}^{1, p}\left(\rho_{j}\right) .
$$

Proof. Fix $\mu \in \mathcal{P}^{1, p}\left(\left(\mathbb{R}^{d}\right)^{N}\right)$. By Theorem 3.2 and Lemma 3.1 we have

$$
\left|\nabla\left(\mu \iota_{j}\right)^{\frac{1}{p}}\left(x_{j}\right)\right|^{p}=\frac{1}{p^{p}} \mu \iota_{j}\left(x_{j}\right)^{1-p}\left|\nabla \mu \iota_{j}\left(x_{j}\right)\right|^{p} \leq \int\left|\nabla_{x_{j}} \mu^{\frac{1}{p}}(X)\right|^{p} \mathrm{~d} \hat{X}_{j} .
$$

Summing on $j$ and recalling the condition (2.3) we get the thesis. As for the second statement, due to the first one clearly we have

$$
\inf \left\{\mathcal{E}^{1, p}(\mu) \mid \mu \in \Pi\left(\rho_{1}, \ldots, \rho_{N}\right)\right\} \geq \sum_{j=1}^{N} \mathcal{E}^{1, p}\left(\rho_{j}\right) .
$$

Let however $\mu(X):=\rho_{1}\left(x_{1}\right) \cdots \rho_{N}\left(x_{N}\right)$; then $\mu$ is such that $\mu \in \mathcal{P}^{1, p}\left(\left(\mathbb{R}^{d}\right)^{N}\right)$ and

$$
\nabla_{x_{j}} \mu^{\frac{1}{p}}=\nabla \rho_{j}^{\frac{1}{p}} \prod_{\substack{k=1 \\ k \neq j}}^{N} \rho_{k}\left(x_{k}\right)^{\frac{1}{p}}
$$

hence

$$
\int\left|\nabla_{x_{j}} \mu^{\frac{1}{p}}(X)\right|^{p} \mathrm{~d} X=\int\left|\nabla \rho_{j}^{\frac{1}{p}}\left(x_{j}\right)\right|^{p} \mathrm{~d} x_{j}=\mathcal{E}^{1, p}\left(\rho_{j}\right) .
$$

Finally summing on $j$ and taking into account the usual condition (2.3),

$$
\mathcal{E}^{1, p}(\mu)=\sum_{j=1}^{N} \mathcal{E}^{1, p}\left(\rho_{j}\right) .
$$


Proposition 4.2. Let $\eta \in C^{\infty}\left(\mathbb{R}^{m}\right), \eta \geq 0$ such that $\int \eta=1$ and define for $\varepsilon>0$

$$
\eta^{\varepsilon}(x)=\frac{1}{\varepsilon^{m}} \eta\left(\frac{x}{\varepsilon}\right) \text {. }
$$

Then, for every $\mu \in \mathcal{P}^{1, p}\left(\mathbb{R}^{m}\right)$,

$$
\mathcal{E}^{1, p}\left(\mu * \eta^{\varepsilon}\right) \leq \mathcal{E}^{1, p}(\mu) \quad \text { and } \quad \lim _{\varepsilon \rightarrow 0} \mathcal{E}^{1, p}\left(\mu * \eta^{\varepsilon}\right)=\mathcal{E}^{1, p}(\mu) .
$$

Proof. By the Hölder inequality with exponents $p$ and $\frac{p}{p-1}$ we have

$$
\begin{aligned}
\left|\nabla\left(\mu * \eta^{\varepsilon}\right)(x)\right| & =\left|\left((\nabla \mu) * \eta^{\varepsilon}\right)(x)\right| \\
& \leq \int|\nabla \mu(y)| \eta^{\varepsilon}(x-y) \mathrm{d} y \\
& \leq\left(\int \mu(y)^{1-p}|\nabla \mu(y)|^{p} \eta^{\varepsilon}(x-y) \mathrm{d} y\right)^{\frac{1}{p}}\left(\mu * \eta^{\varepsilon}\right)(x)^{\frac{p-1}{p}} .
\end{aligned}
$$

Since $\mu * \eta^{\varepsilon} \in C^{\infty}\left(\mathbb{R}^{m}\right)$ we have

whence

$$
\begin{aligned}
\left|\nabla\left(\mu * \eta^{\varepsilon}\right)(x)^{\frac{1}{p}}\right| & =\frac{1}{p}\left(\mu * \eta^{\varepsilon}\right)(x)^{\frac{1-p}{p}}\left|\nabla\left(\mu * \eta^{\varepsilon}\right)(x)\right| \\
& \leq \frac{1}{p}\left(\int \mu(y)^{1-p}|\nabla \mu(y)|^{p} \eta^{\varepsilon}(x-y) \mathrm{d} y\right)^{\frac{1}{p}},
\end{aligned}
$$

$$
\begin{aligned}
\mathcal{E}^{1, p}\left(\mu * \eta^{\varepsilon}\right) & =\int\left|\nabla\left(\mu * \eta^{\varepsilon}\right)^{\frac{1}{p}}(x)\right|^{p} \mathrm{~d} x \\
& \leq \frac{1}{p^{p}} \int \mu(y)^{1-p}|\nabla \mu(y)|^{p} \eta^{\varepsilon}(x-y) \mathrm{d} y \mathrm{~d} x \\
& =\int\left|\nabla \mu^{\frac{1}{p}}(y)\right|^{p} \mathrm{~d} y=\mathcal{E}^{1, p}(\mu) .
\end{aligned}
$$

In order to prove the second part, it suffices to show that $\left(\mu * \eta^{\varepsilon}\right)^{\frac{1}{p}}$ converges strongly to $\mu^{\frac{1}{p}}$ in $W^{1, p}$ to get that

$$
\lim _{\varepsilon \rightarrow 0} \mathcal{E}^{1, p}\left(\mu * \eta^{\varepsilon}\right)=\lim _{\varepsilon \rightarrow 0}\left\|\left(\mu * \eta^{\varepsilon}\right)^{\frac{1}{p}}\right\|_{W^{1, p}}^{p}-1=\left\|\mu^{\frac{1}{p}}\right\|_{W^{1, p}}-1=\mathcal{E}^{1, p}(\mu) .
$$

Since $\left(\mu^{1-p}|\nabla \mu|^{p}\right) * \eta^{\varepsilon} \longrightarrow \mu^{1-p}|\nabla \mu|^{p}$ pointwise a.e., inequality (4.2) gives a domination which allows to conclude thanks to Proposition 2.4.

\section{Definition OF the SMOOTHING OPERATOR}

In this section we start to deal with the main problem of the paper, which we recall here.

Problem: Given $\rho_{1}, \ldots, \rho_{N} \in \mathcal{P}^{1, p}\left(\mathbb{R}^{d}\right)$, and given $\mu \in \Pi\left(\rho_{1}, \ldots, \rho_{N}\right)$, find a family $\left(\mu^{\varepsilon}\right)_{\varepsilon>0}$ such that:

(i) $\mu^{\varepsilon} \in \Pi\left(\rho_{1}, \ldots, \rho_{N}\right)$;

(ii) $\mu^{\varepsilon} \in \mathcal{P}^{1, p}\left(\left(\mathbb{R}^{d}\right)^{N}\right)$;

(iii) $\mu^{\varepsilon} \rightarrow \mu$ as $\varepsilon \rightarrow 0$.

To this end, we will define an operator

$$
\begin{aligned}
\Theta: \mathbb{R}^{+} \times \mathcal{P}\left(\left(\mathbb{R}^{d}\right)^{N}\right) & \longrightarrow \mathcal{P}\left(\left(\mathbb{R}^{d}\right)^{N}\right) \\
(\varepsilon, \mu) & \longmapsto \Theta^{\varepsilon}[\mu]
\end{aligned}
$$

such that:

A. for every $\varepsilon>0$, for every $j=1, \ldots, N$,

$$
\Theta^{\varepsilon}[\mu] L_{j}=\mu L_{j}
$$


B. if $\mu \iota_{j} \in \mathcal{P}^{1, p}\left(\mathbb{R}^{d}\right)$ for evey $j=1, \ldots, N$, then

$$
\Theta^{\varepsilon}[\mu] \in \mathcal{P}^{1, p}\left(\left(\mathbb{R}^{d}\right)^{N}\right) ;
$$

C. for every $\phi \in C_{b}\left(\left(\mathbb{R}^{d}\right)^{N}\right)$,

$$
\lim _{\varepsilon \rightarrow 0} \int \phi \mathrm{d} \Theta^{\varepsilon}[\mu]=\int \phi \mathrm{d} \mu .
$$

This will give a universal construction which solves the problem: properties A C ensure that, taking $\mu^{\varepsilon}:=\Theta^{\varepsilon}[\mu]$, the requirements (i)-(iii) above are satisfied. Moreover, the smoothing operator $\Theta$ will also satisfy the following form of continuity with respect to the measure argument.

Theorem 5.1. Let $\mu^{n}, \mu \in \mathcal{P}\left(\left(\mathbb{R}^{d}\right)^{N}\right)$ such that:

(i) $\mu^{n} \rightarrow \mu$ in duality with $C_{b}\left(\left(\mathbb{R}^{d}\right)^{N}\right)$;

(ii) for every $j=1, \ldots, N,\left.\mu^{n}\right|_{j} \in \mathcal{P}^{1, p}\left(\mathbb{R}^{d}\right)$ and $\left.\mu\right|_{j} \in \mathcal{P}^{1, p}\left(\mathbb{R}^{d}\right)$, with

$$
\lim _{n \rightarrow \infty} d^{1, p}\left(\mu^{n} \iota_{j}, \mu \iota_{j}\right)=0 \text {. }
$$

Then, for every $\varepsilon>0$,

$$
\lim _{n \rightarrow \infty} d^{1, p}\left(\Theta^{\varepsilon}\left[\mu^{n}\right], \Theta^{\varepsilon}[\mu]\right)=0 .
$$

The proof of Theorem 5.1 will be presented in Section 8 .

Now we proceed with the construction of the smoothing operator $\Theta$. Given $\varepsilon>0$, let $\eta^{\varepsilon}: \mathbb{R}^{d} \rightarrow \mathbb{R}^{+}$be

$$
\eta^{\varepsilon}(z)=\frac{1}{(2 \pi \varepsilon)^{d / 2}} \exp \left(-\frac{|z|^{2}}{2 \varepsilon}\right)
$$

For $\mu \in \mathcal{P}\left(\left(\mathbb{R}^{d}\right)^{N}\right)$, we define the measure $\Lambda^{\varepsilon}[\mu]$ as the convolution of $\mu$ with the kernel $\eta^{\varepsilon}\left(x_{1}\right) \cdots \eta^{\varepsilon}\left(x_{N}\right)$, i.e., if $\psi:\left(\mathbb{R}^{d}\right)^{N} \rightarrow \mathbb{R}$ is any continuous bounded function,

$$
\int \psi(Y) \mathrm{d} \Lambda^{\varepsilon}[\mu](Y):=\iint \psi(Y) \prod_{k=1}^{N} \eta^{\varepsilon}\left(y_{k}-x_{k}\right) \mathrm{d} \mu(X) \mathrm{d} Y .
$$

Notice that $\Lambda^{\varepsilon}[\mu]$ is absolutely continuous with respect to the Lebesgue measure, with density

$$
\Lambda^{\varepsilon}[\mu](Y)=\int \prod_{k=1}^{N} \eta^{\varepsilon}\left(y_{k}-x_{k}\right) \mathrm{d} \mu(X) .
$$

Finally, if $\psi:\left(\mathbb{R}^{d}\right)^{N} \rightarrow \mathbb{R}$ is any continuous bounded function, we define $\Theta^{\varepsilon}[\mu]$ via the expression

$$
\int \psi(X) \mathrm{d} \Theta^{\varepsilon}[\mu](X):=\left.\iint \psi(X) \prod_{k=1}^{N} \frac{\eta^{\varepsilon}\left(y_{k}-x_{k}\right)}{\left(\left.\mu\right|_{k} * \eta^{\varepsilon}\right)\left(y_{k}\right)} \mathrm{d} \mu\right|_{k}\left(x_{k}\right) \mathrm{d} \Lambda^{\varepsilon}[\mu](Y) .
$$

Here, with a slight abuse of notation, the denominator $\left(\mu L_{k} * \eta^{\varepsilon}\right)\left(y_{k}\right)$ denotes the density of the measure $\left.\mu\right|_{k} * \eta^{\varepsilon}$ evaluated at $y_{k}$, namely

$$
\left(\mu \iota_{k} * \eta^{\varepsilon}\right)\left(y_{k}\right)=\int \eta^{\varepsilon}\left(y_{k}-x_{k}\right) \mathrm{d} \mu \iota_{k}\left(x_{k}\right),
$$

and is always strictly positive, since $\left.\mu\right|_{k}$ is a probability and $\eta^{\varepsilon}>0$. 
Remark 2. This construction fits into the general framework for the composition of transport plans, as in [2, Section 5.3]. Indeed, the definition of $\Theta^{\varepsilon}[\mu]$ may be seen as follows: as a first step we regularize $\mu$ by convolution; secondly, we consider the 2-transport plans $\beta_{j}$ for $j=1, \ldots, N$ defined by

$$
\int \phi(x, y) \mathrm{d} \beta_{j}(x, y)=\left.\int \phi(x, y) \eta^{\varepsilon}(x-y) \mathrm{d} \mu\right|_{j}(y) \mathrm{d} y
$$

for any $\phi \in C_{b}\left(\mathbb{R}^{d} \times \mathbb{R}^{d}\right)$. Notice that $\beta_{j}$ has marginals $\mu \iota_{j} * \eta^{\varepsilon}$ and $\mu \iota_{j}$. Then $\Theta^{\varepsilon}[\mu]$ corresponds to the composition of $\Lambda^{\varepsilon}[\mu]$ with $\beta_{j}$ on each corresponding $j$-th marginal.

Lemma 5.2 (Property $j=1, \ldots, N$ the following hold.

(i) $\left.\Lambda^{\varepsilon}[\mu]\right|_{j}=\left.\mu\right|_{j} * \eta^{\varepsilon}$

(ii) $\Theta^{\varepsilon}[\mu] L_{j}=\mu L_{j}$.

Proof. (i) If $\phi \in C_{b}\left(\mathbb{R}^{d}\right)$, by the Fubini's Theorem we have

$$
\begin{aligned}
\int \phi\left(y_{j}\right) \mathrm{d} \Lambda^{\varepsilon}[\mu] L_{j}\left(y_{j}\right) & =\int \phi\left(y_{j}\right) \mathrm{d} \Lambda^{\varepsilon}[\mu](Y) \\
& =\int \phi\left(y_{j}\right) \prod_{k=1}^{N} \eta^{\varepsilon}\left(y_{k}-x_{k}\right) \mathrm{d} \mu(X) \mathrm{d} Y \\
& =\int \phi\left(y_{j}\right) \eta^{\varepsilon}\left(y_{j}-x_{j}\right) \mathrm{d} \mu(X) \mathrm{d} y_{j} \\
& =\int \phi\left(y_{j}\right) \eta^{\varepsilon}\left(y_{j}-x_{j}\right) \mathrm{d} \mu \iota_{j}\left(x_{j}\right)=\int \phi\left(y_{j}\right) \mathrm{d}\left(\mu L_{j} * \eta^{\varepsilon}\right)\left(y_{j}\right) .
\end{aligned}
$$

(ii) Using (i), if $\phi \in C_{b}\left(\mathbb{R}^{d}\right)$, by the Fubini's Theorem we have

$$
\begin{aligned}
& \int \phi\left(x_{j}\right) \mathrm{d}\left(\left.\Theta^{\varepsilon}[\mu]\right|_{j}\right)(X)=\int \phi\left(x_{j}\right) \mathrm{d}\left(\Theta^{\varepsilon}[\mu]\right)(X) \\
& =\iint \phi\left(x_{j}\right) \prod_{k=1}^{N} \frac{\eta^{\varepsilon}\left(y_{k}-x_{k}\right)}{\left(\mu \iota_{k} * \eta^{\varepsilon}\right)\left(y_{k}\right)} \mathrm{d} \mu \iota_{k}\left(x_{k}\right) \mathrm{d} \Lambda^{\varepsilon}[\mu](Y) \\
& =\int \phi\left(x_{j}\right) \frac{\eta^{\varepsilon}\left(y_{j}-x_{j}\right)}{\left(\mu \iota_{j} * \eta^{\varepsilon}\right)\left(y_{j}\right)} \mathrm{d} \Lambda^{\varepsilon}[\mu](Y) \mathrm{d} \mu\left\llcorner_{j}\left(x_{j}\right)\right. \\
& =\left.\int \phi\left(x_{j}\right) \frac{\eta^{\varepsilon}\left(y_{j}-x_{j}\right)}{\left(\mu \iota_{j} * \eta^{\varepsilon}\right)\left(y_{j}\right)} \mathrm{d}\left(\mu \iota_{j} * \eta^{\varepsilon}\right)\left(y_{j}\right) \mathrm{d} \mu\right|_{j}\left(x_{j}\right) \\
& =\int \phi\left(x_{j}\right) \mathrm{d} \mu \iota_{j}\left(x_{j}\right) \text {. }
\end{aligned}
$$

\section{Regularity of $\Theta$}

In this Section we prove that $\Theta$ satisfies property[B] of Section 5 , Moreover, some additional estimates on the $W^{1, p}$-energy of $\Theta^{\varepsilon}[\mu]$ also hold. Let $\mu \in \mathcal{P}\left(\left(\mathbb{R}^{d}\right)^{N}\right)$ such that $\mu l_{j} \in \mathcal{P}^{1, p}\left(\mathbb{R}^{d}\right)$ for every $j=1, \ldots, N$. Then $\Theta^{\varepsilon}[\mu]$ is absolutely continuous with respect to the Lebesgue measure, with density given by

$$
\Theta^{\varepsilon}[\mu](X)=\int P^{\varepsilon}[\mu](X, Y) \mathrm{d} Y
$$

where we denote by $P^{\varepsilon}[\mu]$ the integral kernel appearing in (15.2), namely

$$
P^{\varepsilon}[\mu](X, Y):=\prod_{k=1}^{N} \frac{\eta^{\varepsilon}\left(y_{k}-x_{k}\right)}{\left(\mu \iota_{k} * \eta^{\varepsilon}\right)\left(y_{k}\right)} \mu \iota_{k}\left(x_{k}\right) \Lambda^{\varepsilon}[\mu](Y) .
$$


Let us denote by

$$
\begin{aligned}
\nabla_{x_{j}} \Theta^{\varepsilon}[\mu](X):= & \frac{\left.\nabla \mu\right|_{j}\left(x_{j}\right)}{\mu \iota_{j}\left(x_{j}\right)} \Theta^{\varepsilon}[\mu](X) \\
& -\int \frac{\nabla \eta^{\varepsilon}\left(y_{j}-x_{j}\right)}{\eta^{\varepsilon}\left(y_{j}-x_{j}\right)} P^{\varepsilon}[\mu](X, Y) \mathrm{d} Y .
\end{aligned}
$$

We claim that $\nabla_{x_{j}} \Theta^{\varepsilon}[\mu](X)$ is the weak gradient with respect to the $j$-th variable of $\Theta^{\varepsilon}[\mu](X)$ in $W^{1,1}\left(\left(\mathbb{R}^{d}\right)^{N}\right)$. Indeed, if $\psi \in C_{c}^{\infty}\left(\left(\mathbb{R}^{d}\right)^{N}\right)$, by the Fubini's Theorem we may perform first the integration in $x_{j}$ to get

$$
\begin{aligned}
\int \nabla_{x_{j}} \psi(X) \Theta^{\varepsilon}[\mu](X) \mathrm{d} X= & \iint \nabla_{x_{j}} \psi(X) P^{\varepsilon}[\mu](X, Y) \mathrm{d} X \mathrm{~d} Y \\
= & \iint \psi(X) \frac{\left.\nabla \mu\right|_{j}\left(x_{j}\right)}{\mu \iota_{j}\left(x_{j}\right)} P^{\varepsilon}[\mu](X, Y) \mathrm{d} X \mathrm{~d} Y \\
& -\iint \psi(X) \frac{\nabla \eta^{\varepsilon}\left(y_{j}-x_{j}\right)}{\eta^{\varepsilon}\left(y_{j}-x_{j}\right)} P^{\varepsilon}[\mu](X, Y) \mathrm{d} X \mathrm{~d} Y \\
= & \int \psi(X) \frac{\left.\nabla \mu\right|_{j}\left(x_{j}\right)}{\left.\mu\right|_{j}\left(x_{j}\right)} \Theta^{\varepsilon}[\mu](X) \mathrm{d} X \\
& -\int \psi(X) \int \frac{\nabla \eta^{\varepsilon}\left(y_{j}-x_{j}\right)}{\eta^{\varepsilon}\left(y_{j}-x_{j}\right)} P^{\varepsilon}[\mu](X, Y) \mathrm{d} Y \mathrm{~d} X .
\end{aligned}
$$

To conclude that $\Theta^{\varepsilon}[\mu] \in \mathcal{P}^{1, p}\left(\left(\mathbb{R}^{d}\right)^{N}\right)$, in view of Proposition 2.3, it suffices to show a suitable domination, which is given by the following

Lemma 6.1. Let $\mu \in \mathcal{P}\left(\left(\mathbb{R}^{d}\right)^{N}\right)$ such that $\mu L_{j} \in \mathcal{P}^{1, p}\left(\mathbb{R}^{d}\right)$ for every $j=1, \ldots, N$. Then

$$
\begin{aligned}
& \left|\nabla_{x_{j}} \Theta^{\varepsilon}[\mu](X)\right|^{p} \Theta^{\varepsilon}[\mu](X)^{1-p} \\
& \leq 2^{p-1}\left(\frac{\left|\nabla \mu L_{j}\left(x_{j}\right)\right|^{p}}{\mu L_{j}\left(x_{j}\right)^{p}} \Theta^{\varepsilon}[\mu](X)+\int \frac{\left|\nabla \eta^{\varepsilon}\left(y_{j}-x_{j}\right)\right|^{p}}{\eta^{\varepsilon}\left(y_{j}-x_{j}\right)^{p}} P^{\varepsilon}[\mu](X, Y) \mathrm{d} Y\right)
\end{aligned}
$$

Proof. By the triangular inequality we immediately get

$$
\left|\nabla_{x_{j}} \Theta^{\varepsilon}[\mu](X)\right| \leq \frac{\left|\nabla \mu \iota_{j}\left(x_{j}\right)\right|}{\mu\left\llcorner_{j}\left(x_{j}\right)\right.} \Theta^{\varepsilon}[\mu](X)+\int \frac{\left|\nabla \eta^{\varepsilon}\left(y_{j}-x_{j}\right)\right|}{\eta^{\varepsilon}\left(y_{j}-x_{j}\right)} P^{\varepsilon}[\mu](X, Y) \mathrm{d} Y .
$$

Using the Hölder inequality with exponents $p$ and $\frac{p}{p-1}$,

$$
\begin{aligned}
& \int \frac{\left|\nabla \eta^{\varepsilon}\left(y_{j}-x_{j}\right)\right|}{\eta^{\varepsilon}\left(y_{j}-x_{j}\right)} P^{\varepsilon}[\mu](X, Y) \mathrm{d} Y \\
& \leq\left(\int \frac{\left|\nabla \eta^{\varepsilon}\left(y_{j}-x_{j}\right)\right|^{p}}{\eta^{\varepsilon}\left(y_{j}-x_{j}\right)^{p}} P^{\varepsilon}[\mu](X, Y) \mathrm{d} Y\right)^{\frac{1}{p}} \Theta^{\varepsilon}[\mu](X)^{\frac{p-1}{p}}
\end{aligned}
$$

and the thesis follows.

Finally we get the proof of property B, together with the usual explicit formula for the weak gradient of $\Theta^{\varepsilon}[\mu]^{\frac{1}{p}}$.

Theorem 6.2 (Property $\mathrm{B})$. Let $\mu \in \mathcal{P}\left(\left(\mathbb{R}^{d}\right)^{N}\right)$ such that $\left.\mu\right|_{j} \in \mathcal{P}^{1, p}\left(\mathbb{R}^{d}\right)$ for every $j=1, \ldots, N$. Then $\Theta^{\varepsilon}[\mu] \in \mathcal{P}^{1, p}\left(\left(\mathbb{R}^{d}\right)^{N}\right)$, and

$$
\nabla_{x_{j}} \Theta^{\varepsilon}[\mu]^{\frac{1}{p}}(X)=\frac{1}{p} \Theta^{\varepsilon}[\mu](X)^{\frac{1-p}{p}} \nabla_{x_{j}} \Theta^{\varepsilon}[\mu](X) .
$$


Proof. Recalling Proposition 2.3, it suffices to check that condition (2.4) holds. Using Lemma 6.1 we have

$$
\begin{aligned}
& \int\left|\nabla_{x_{j}} \Theta^{\varepsilon}[\mu](X)\right|^{p} \Theta^{\varepsilon}[\mu](X)^{1-p} \mathrm{~d} X \\
& \leq 2^{p-1}\left(\int \frac{\left|\nabla \mu L_{j}\left(x_{j}\right)\right|^{p}}{\mu L_{j}\left(x_{j}\right)^{p}} \Theta^{\varepsilon}[\mu](X) \mathrm{d} X+\iint \frac{\left|\nabla \eta^{\varepsilon}\left(y_{j}-x_{j}\right)\right|^{p}}{\eta^{\varepsilon}\left(y_{j}-x_{j}\right)^{p}} P^{\varepsilon}[\mu](X, Y) \mathrm{d} Y \mathrm{~d} X\right) \\
& =2^{p-1}\left(\int \frac{\left|\nabla \mu L_{j}\left(x_{j}\right)\right|^{p}}{\mu L_{j}\left(x_{j}\right)^{p-1}} \mathrm{~d} x_{j}+\int \frac{\left|\nabla \eta^{\varepsilon}(z)\right|^{p}}{\eta^{\varepsilon}(z)^{p-1}} \mathrm{~d} z\right) \\
& =2^{p-1} p^{p}\left\|\nabla\left(\mu L_{j}\right)^{\frac{1}{p}}\right\|_{p}^{p}+C(d, \varepsilon, p),
\end{aligned}
$$

where the latter is a constant depending only on the dimension $d$, the exponent $p$ and $\varepsilon$.

From Theorem 6.2 we get also some estimates on the $W^{1, p}$-energy of $\Theta^{\varepsilon}[\mu]$. In the case $p=2$ the Hilbertian structure allows to simplify some computation and to get sharper constants.

Theorem 6.3. Let $\mu \in \mathcal{P}\left(\left(\mathbb{R}^{d}\right)^{N}\right)$ such that $\mu\left\llcorner_{j} \in \mathcal{P}^{1,2}\left(\mathbb{R}^{d}\right)\right.$ for every $j=1, \ldots, N$.

Then

$$
\mathcal{E}^{1,2}\left(\Theta^{\varepsilon}[\mu]\right) \leq \sum_{j=1}^{N} \mathcal{E}^{1,2}\left(\mu\left\llcorner_{j}\right)+\frac{N c(d)}{\varepsilon}\right.
$$

where $c(d)$ is a constant depending only on the dimension $d$.

If in addition $\mu \in \mathcal{P}^{1,2}\left(\left(\mathbb{R}^{d}\right)^{N}\right)$, then

$$
\mathcal{E}^{1,2}\left(\Theta^{\varepsilon}[\mu]\right) \leq \sum_{j=1}^{N}\left(\left\|\nabla_{x_{j}} \sqrt{\Lambda^{\varepsilon}[\mu]}\right\|_{2}+\Delta(\varepsilon, \mu)\right)^{2} .
$$

where

$$
\Delta(\varepsilon, \mu)=\sqrt{\mathcal{E}^{1,2}\left(\left.\mu\right|_{j}\right)-\mathcal{E}^{1,2}\left(\mu \iota_{j} * \eta^{\varepsilon}\right)} .
$$

Proof of Theorem 6.3. By Theorem 6.2 we have

$$
\begin{aligned}
\mathcal{E}^{1,2}\left(\Theta^{\varepsilon}[\mu]\right)= & \int\left|\nabla \sqrt{\Theta^{\varepsilon}[\mu]}(X)\right|^{2} \mathrm{~d} X \\
= & \frac{1}{4} \int \frac{\left|\nabla \Theta^{\varepsilon}[\mu](X)\right|^{2}}{\Theta^{\varepsilon}[\mu](X)} \mathrm{d} X \\
= & \frac{1}{4} \sum_{j=1}^{N} \int \frac{\left|\nabla \mu \iota_{j}\left(x_{j}\right)\right|^{2}}{\mu \iota_{j}\left(x_{j}\right)^{2}} \Theta^{\varepsilon}[\mu](X) \mathrm{d} X \\
& -\sum_{j=1}^{N} \iint \frac{\nabla \mu \iota_{j}\left(x_{j}\right) \cdot \nabla \eta^{\varepsilon}\left(y_{j}-x_{j}\right)}{\mu \downarrow_{j}\left(x_{j}\right) \eta^{\varepsilon}\left(y_{j}-x_{j}\right)} P^{\varepsilon}[\mu](X, Y) \mathrm{d} X \mathrm{~d} Y \\
& +\sum_{j=1}^{N} \int \frac{1}{\Theta^{\varepsilon}[\mu](X)}\left|\int \frac{\nabla \eta^{\varepsilon}\left(y_{j}-x_{j}\right)}{\eta^{\varepsilon}\left(y_{j}-x_{j}\right)} P^{\varepsilon}[\mu](X, Y) \mathrm{d} Y\right|^{2} \mathrm{~d} X .
\end{aligned}
$$


We treat the three terms in order. First we have

$$
\begin{aligned}
\frac{1}{4} \sum_{j=1}^{N} \int \frac{\left|\nabla \mu \iota_{j}\left(x_{j}\right)\right|^{2}}{\mu \iota_{j}\left(x_{j}\right)^{2}} \Theta^{\varepsilon}[\mu](X) \mathrm{d} X & =\frac{1}{4} \sum_{j=1}^{N} \int \frac{\mid \nabla \mu\left\llcorner\left._{j}\left(x_{j}\right)\right|^{2}\right.}{\mu \iota_{j}\left(x_{j}\right)} \mathrm{d} x_{j} \\
& =\sum_{j=1}^{N} \int\left|\nabla \sqrt{\mu \iota_{j}}\left(x_{j}\right)\right|^{2} \mathrm{~d} x_{j} \\
& =\sum_{j=1}^{N} \mathcal{E}^{1,2}\left(\mu \iota_{j}\right) .
\end{aligned}
$$

The middle term vanishes. Indeed, using Fubini's theorem and a change of variables,

$$
\begin{aligned}
& \iint \frac{\nabla \mu\left\llcorner_{j}\left(x_{j}\right) \cdot \nabla \eta^{\varepsilon}\left(y_{j}-x_{j}\right)\right.}{\mu \iota_{j}\left(x_{j}\right) \eta^{\varepsilon}\left(y_{j}-x_{j}\right)} P^{\varepsilon}[\mu](X, Y) \mathrm{d} X \mathrm{~d} Y \\
& =\iint \frac{\nabla \mu\left\llcorner_{j}\left(x_{j}\right) \cdot \nabla \eta^{\varepsilon}\left(y_{j}-x_{j}\right)\right.}{\mu \iota_{j}\left(x_{j}\right) \eta^{\varepsilon}\left(y_{j}-x_{j}\right)} \frac{\eta^{\varepsilon}\left(y_{j}-x_{j}\right)}{\left(\mu \iota_{j} * \eta^{\varepsilon}\right)\left(y_{j}\right)} \mu \downarrow_{j}\left(x_{j}\right) \Lambda^{\varepsilon}[\mu](Y) \mathrm{d} x_{j} \mathrm{~d} Y \\
& =\iint \nabla \mu\left\llcorner_{j}\left(x_{j}\right) \cdot \nabla \eta^{\varepsilon}\left(y_{j}-x_{j}\right) \mathrm{d} x_{j} \mathrm{~d} y_{j}\right. \\
& =\left(\iint \nabla \mu\left\llcorner_{j}\left(x_{j}\right) \mathrm{d} x_{j}\right) \cdot\left(\int \nabla \eta^{\varepsilon}(z) \mathrm{d} z\right)\right.
\end{aligned}
$$

and the second term is zero, as it can be seen, for instance, integrating in spherical coordinates.

Finally, by the Cauchy-Schwarz inequality,

$$
\begin{aligned}
& \left|\int \frac{\nabla \eta^{\varepsilon}\left(y_{j}-x_{j}\right)}{\eta^{\varepsilon}\left(y_{j}-x_{j}\right)} P^{\varepsilon}[\mu](X, Y) \mathrm{d} Y\right|^{2} \\
& \leq\left(\int \frac{\left|\nabla \eta^{\varepsilon}\left(y_{j}-x_{j}\right)\right|^{2}}{\eta^{\varepsilon}\left(y_{j}-x_{j}\right)^{2}} P^{\varepsilon}[\mu](X, Y) \mathrm{d} Y\right) \int P^{\varepsilon}[\mu](X, Y) \mathrm{d} Y \\
& =\Theta^{\varepsilon}[\mu](X) \int \frac{\left|\nabla \eta^{\varepsilon}\left(y_{j}-x_{j}\right)\right|^{2}}{\eta^{\varepsilon}\left(y_{j}-x_{j}\right)^{2}} P^{\varepsilon}[\mu](X, Y) \mathrm{d} Y .
\end{aligned}
$$

Hence the third term is bounded by

$$
\begin{aligned}
& \sum_{j=1}^{N} \iint \frac{\left|\nabla \eta^{\varepsilon}\left(y_{j}-x_{j}\right)\right|^{2}}{\eta^{\varepsilon}\left(y_{j}-x_{j}\right)^{2}} P^{\varepsilon}[\mu](X, Y) \mathrm{d} Y \mathrm{~d} X \\
& =\sum_{j=1}^{N} \iint \frac{\left|\nabla \eta^{\varepsilon}\left(y_{j}-x_{j}\right)\right|^{2}}{\eta^{\varepsilon}\left(y_{j}-x_{j}\right)} \mu \iota_{j}\left(x_{j}\right) \Lambda^{\varepsilon}[\mu](Y) \mathrm{d} Y \mathrm{~d} x_{j} \\
& =\sum_{j=1}^{N} \iint \frac{\left|\nabla \eta^{\varepsilon}\left(y_{j}-x_{j}\right)\right|^{2}}{\eta^{\varepsilon}\left(y_{j}-x_{j}\right)} \mu \iota_{j}\left(x_{j}\right) \mathrm{d} y_{j} \mathrm{~d} x_{j} \\
& =N \int \frac{\left|\nabla \eta^{\varepsilon}(z)\right|^{2}}{\eta^{\varepsilon}(z)} \mathrm{d} z=\frac{N c(d)}{\varepsilon},
\end{aligned}
$$

where $c(d)$ is a constant depending only on the dimension $d$. 
In order to show the second part of the statement, notice that, if $\mu$ is $W^{1, p_{-}}$ regular, performing a change of variables in (6.2) we may write

$$
\begin{aligned}
\nabla_{x_{j}} \Theta^{\varepsilon}[\mu](X)= & \int\left(\frac{\nabla \mu \iota_{j}\left(x_{j}\right)}{\mu \iota_{j}\left(x_{j}\right)}-\frac{\nabla\left(\mu \iota_{j} * \eta^{\varepsilon}\right)\left(y_{j}\right)}{\left(\mu\left\llcorner_{j} * \eta^{\varepsilon}\right)\left(y_{j}\right)\right.}\right) P^{\varepsilon}[\mu](X, Y) \mathrm{d} Y \\
& +\int \nabla_{x_{j}} \Lambda^{\varepsilon}[\mu](Y) \prod_{j=1}^{N} \frac{\eta^{\varepsilon}\left(y_{j}-x_{j}\right)}{\left(\mu \iota_{j} * \eta^{\varepsilon}\right)\left(y_{j}\right)} \mu \downarrow_{j}\left(x_{j}\right) \mathrm{d} Y \\
= & : I(X)+I I(X)
\end{aligned}
$$

We estimate both terms via the Cauchy-Schwarz inequality to get

$$
\begin{aligned}
& |I(X)|^{2} \leq\left(\int\left|\frac{\left.\nabla \mu\right|_{j}\left(x_{j}\right)}{\mu \iota_{j}\left(x_{j}\right)}-\frac{\nabla\left(\mu\left\llcorner_{j} * \eta^{\varepsilon}\right)\left(y_{j}\right)\right.}{\left(\left.\mu\right|_{j} * \eta^{\varepsilon}\right)\left(y_{j}\right)}\right|^{2} P^{\varepsilon}[\mu](X, Y) \mathrm{d} Y\right) \int P^{\varepsilon}[\mu](X, Y) \mathrm{d} Y \\
& =\Theta^{\varepsilon}[\mu](X) \int\left|\frac{\nabla \mu\left\llcorner_{j}\left(x_{j}\right)\right.}{\mu \iota_{j}\left(x_{j}\right)}-\frac{\nabla\left(\mu\left\llcorner_{j} * \eta^{\varepsilon}\right)\left(y_{j}\right)\right.}{\left(\mu \iota_{j} * \eta^{\varepsilon}\right)\left(y_{j}\right)}\right|^{2} P^{\varepsilon}[\mu](X, Y) \mathrm{d} Y
\end{aligned}
$$

and

$$
\begin{aligned}
|I I(X)|^{2} & \leq\left(\int \frac{\left|\nabla_{x_{j}} \Lambda^{\varepsilon}[\mu](Y)\right|^{2}}{\Lambda^{\varepsilon}[\mu](Y)^{2}} P^{\varepsilon}[\mu](X, Y) \mathrm{d} Y\right) \int P^{\varepsilon}[\mu](X, Y) \mathrm{d} Y \\
& =\Theta^{\varepsilon}[\mu](X) \int \frac{\left|\nabla_{x_{j}} \Lambda^{\varepsilon}[\mu](Y)\right|^{2}}{\Lambda^{\varepsilon}[\mu](Y)^{2}} P^{\varepsilon}[\mu](X, Y) \mathrm{d} Y .
\end{aligned}
$$

It follows that

$$
\begin{aligned}
& \int \frac{|I(X)|^{2}}{\Theta^{\varepsilon}[\mu](X)} \mathrm{d} X \leq \iint\left|\frac{\nabla \mu \downarrow_{j}\left(x_{j}\right)}{\mu \downarrow_{j}\left(x_{j}\right)}-\frac{\nabla\left(\left.\mu\right|_{j} * \eta^{\varepsilon}\right)\left(y_{j}\right)}{\left(\mu \downarrow_{j} * \eta^{\varepsilon}\right)\left(y_{j}\right)}\right|^{2} P^{\varepsilon}[\mu](X, Y) \mathrm{d} X \mathrm{~d} Y \\
& =\iint\left|\frac{\nabla \mu \iota_{j}\left(x_{j}\right)}{\mu \iota_{j}\left(x_{j}\right)}-\frac{\nabla\left(\mu \iota_{j} * \eta^{\varepsilon}\right)\left(y_{j}\right)}{\left(\mu \iota_{j} * \eta^{\varepsilon}\right)\left(y_{j}\right)}\right|^{2} \eta^{\varepsilon}\left(y_{j}-x_{j}\right) \mu \iota_{j}\left(x_{j}\right) \mathrm{d} x_{j} \mathrm{~d} y_{j} \\
& =\int \frac{\mid \nabla \mu\left\llcorner\left._{j}\left(x_{j}\right)\right|^{2}\right.}{\mu \iota_{j}\left(x_{j}\right)} \mathrm{d} x_{j}-\int \frac{\mid \nabla\left(\left.\mu\left\llcorner_{j} * \eta^{\varepsilon}\right)\left(y_{j}\right)\right|^{2}\right.}{\left(\mu \iota_{j} * \eta^{\varepsilon}\right)\left(y_{j}\right)} \mathrm{d} y_{j} \\
& =4 \mathcal{E}^{1,2}\left(\mu \iota_{j}\right)-4 \mathcal{E}^{1,2}\left(\left.\mu\right|_{j} * \eta^{\varepsilon}\right)
\end{aligned}
$$

and

$$
\begin{aligned}
\int \frac{|I(X)|^{2}}{\Theta^{\varepsilon}[\mu](X)} \mathrm{d} X & \leq \iint \frac{\left|\nabla_{x_{j}} \Lambda^{\varepsilon}[\mu](Y)\right|^{2}}{\Lambda^{\varepsilon}[\mu](Y)^{2}} P^{\varepsilon}[\mu](X, Y) \mathrm{d} X \mathrm{~d} Y \\
& =\int \frac{\left|\nabla_{x_{j}} \Lambda^{\varepsilon}[\mu](Y)\right|^{2}}{\Lambda^{\varepsilon}[\mu](Y)} \mathrm{d} Y
\end{aligned}
$$

Hence, for every $\tau_{j}>0$,

$$
\begin{aligned}
& \int\left|\nabla_{x_{j}} \sqrt{\Theta^{\varepsilon}[\mu]}(X)\right|^{2} \mathrm{~d} X \\
& \leq\left(1+\tau_{j}\right) \frac{1}{4} \int \frac{|I(X)|^{2}}{\Theta^{\varepsilon}[\mu](X)} \mathrm{d} X+\left(1+\tau_{j}^{-1}\right) \frac{1}{4} \int \frac{|I I(X)|^{2}}{\Theta^{\varepsilon}[\mu](X)} \mathrm{d} X \\
& \leq\left(1+\tau_{j}\right)\left(\mathcal{E}^{1,2}\left(\left.\mu\right|_{j}\right)-\mathcal{E}^{1,2}\left(\left.\mu\right|_{j} * \eta^{\varepsilon}\right)\right)+\left(1+\tau_{j}^{-1}\right) \int \frac{\left|\nabla_{x_{j}} \Lambda^{\varepsilon}[\mu](Y)\right|^{2}}{4 \Lambda^{\varepsilon}[\mu](Y)} \mathrm{d} Y .
\end{aligned}
$$

Optimizing in $\tau_{j}$ and summing over $j=1, \ldots, N$ we get the thesis. 
Theorem 6.4. Let $\mu \in \mathcal{P}\left(\left(\mathbb{R}^{d}\right)^{N}\right)$. Then there exists a constant $c(d, p)$ depending on the dimension $d$ and the exponent $p$ such that

$$
\mathcal{E}^{1, p}\left(\Theta^{\varepsilon}[\mu]\right) \leq \sum_{j=1}^{N}\left(\mathcal{E}^{1, p}\left(\left.\mu\right|_{j}\right)^{\frac{1}{p}}+\frac{c(d, p)}{\sqrt{\varepsilon}}\right)^{p}
$$

If in addition $\mu \in \mathcal{P}^{1, p}\left(\left(\mathbb{R}^{d}\right)^{N}\right)$ and $p>1$, then

$$
\mathcal{E}^{1, p}\left(\Theta^{\varepsilon}[\mu]\right) \leq \sum_{j=1}^{N}\left(\left\|\nabla_{x_{j}} \Lambda^{\varepsilon}[\mu]^{\frac{1}{p}}\right\|_{p}+c_{p} \Delta(\varepsilon, p, \mu)\right)^{p}
$$

where

$\Delta(\varepsilon, p, \mu)= \begin{cases}{\left[\left(\mathcal{E}^{1, p}\left(\left.\mu\right|_{j}\right)+\mathcal{E}^{1, p}\left(\left.\mu\right|_{j} * \eta^{\varepsilon}\right)\right)^{\frac{1}{p-1}}-2 \mathcal{E}^{1, p}\left(\left.\mu\right|_{j} * \eta^{\varepsilon}\right)^{\frac{1}{p-1}}\right]^{\frac{p-1}{p}}} & 1<p<2 \\ \left(\mathcal{E}^{1, p}\left(\left.\mu\right|_{j}\right)-\mathcal{E}^{1, p}\left(\left.\mu\right|_{j} * \eta^{\varepsilon}\right)\right)^{\frac{1}{p}} & p \geq 2\end{cases}$

and $c_{p}$ is a suitable constant depending only on the exponent $p$.

Proof. Combining Theorem 6.2 and Lemma 6.1 we get the first part of the statement, proceeding as in the proof of Theorem 6.3 and using the triangular inequality in $L^{p}$. When the marginals are regular, we use (6.5) to write $\nabla_{x_{j}} \Theta^{\varepsilon}[\mu](X)=$ $I(X)+I I(X)$, and estimate both terms via the Hölder inequality to get

$$
\begin{aligned}
|I(X)|^{p} & \leq \Theta^{\varepsilon}[\mu](X)^{p-1} \int\left|\frac{\nabla \mu \iota_{j}\left(x_{j}\right)}{\mu \iota_{j}\left(x_{j}\right)}-\frac{\nabla\left(\left.\mu\right|_{j} * \eta^{\varepsilon}\right)\left(y_{j}\right)}{\left(\mu\left\llcorner_{j} * \eta^{\varepsilon}\right)\left(y_{j}\right)\right.}\right|^{p} P^{\varepsilon}[\mu](X, Y) \mathrm{d} Y, \\
|I I(X)|^{p} & \leq \Theta^{\varepsilon}[\mu](X)^{p-1} \int \frac{\left|\nabla_{x_{j}} \Lambda^{\varepsilon}[\mu](Y)\right|^{p}}{\Lambda^{\varepsilon}[\mu](Y)^{p}} P^{\varepsilon}[\mu](X, Y) \mathrm{d} Y .
\end{aligned}
$$

When we integrate with respect to the $X$ variable, the triangular inequality in $L^{p}$ gives

$$
\begin{aligned}
& \left(\int \frac{\left|\nabla_{x_{j}} \Theta^{\varepsilon}[\mu](X)\right|^{p}}{\Theta^{\varepsilon}[\mu](X)^{1-p}} \mathrm{~d} X\right)^{\frac{1}{p}} \\
& \leq\left(\iint\left|\frac{\nabla \mu\left\llcorner_{j}\left(x_{j}\right)\right.}{\mu \iota_{j}\left(x_{j}\right)}-\frac{\nabla\left(\mu\left\llcorner_{j} * \eta^{\varepsilon}\right)\left(y_{j}\right)\right.}{\left(\mu\left\llcorner_{j} * \eta^{\varepsilon}\right)\left(y_{j}\right)\right.}\right|^{p} P^{\varepsilon}[\mu](X, Y) \mathrm{d} Y \mathrm{~d} X\right)^{\frac{1}{p}} \\
& +\left(\iint \frac{\left|\nabla_{x_{j}} \Lambda^{\varepsilon}[\mu](Y)\right|^{p}}{\Lambda^{\varepsilon}[\mu](Y)^{p}} P^{\varepsilon}[\mu](X, Y) \mathrm{d} Y \mathrm{~d} X\right)^{\frac{1}{p}} \\
& =\left(\left.\iint\left|\frac{\nabla \mu \iota_{j}(x)}{\mu \iota_{j}(x)}-\frac{\nabla\left(\mu \iota_{j} * \eta^{\varepsilon}\right)(y)}{\left(\mu \iota_{j} * \eta^{\varepsilon}\right)(y)}\right|^{p} \eta^{\varepsilon}(y-x) \mu\right|_{j}(x) \mathrm{d} x \mathrm{~d} y\right)^{\frac{1}{p}} \\
& +\left(\int \frac{\left|\nabla_{x_{j}} \Lambda^{\varepsilon}[\mu](Y)\right|^{p}}{\Lambda^{\varepsilon}[\mu](Y)^{p-1}} \mathrm{~d} Y\right)^{\frac{1}{p}} .
\end{aligned}
$$

Now we recall the following inequalities by Clarkson [5]: if $f, g \in L^{p}(\nu)$, then

$$
\begin{aligned}
& \left\|\frac{f-g}{2}\right\|^{p} \leq \frac{1}{2}\|f\|^{p}+\frac{1}{2}\|g\|^{p}-\left\|\frac{f+g}{2}\right\|^{p} \quad p \geq 2 \\
& \left\|\frac{f-g}{2}\right\|^{\frac{p}{p-1}} \leq\left(\frac{1}{2}\|f\|^{p}+\frac{1}{2}\|g\|^{p}\right)^{\frac{1}{p-1}}-\left\|\frac{f+g}{2}\right\|^{\frac{p}{p-1}} \quad 1<p<2,
\end{aligned}
$$

where all the norms are $L^{p}(\nu)$ norms. 
If we apply (6.8) on $\mathbb{R}^{d} \times \mathbb{R}^{d}$ with $f(x, y)=\frac{\nabla \mu l_{j}(x)}{\mu l_{j}(x)}, g(x, y)=\frac{\nabla\left(\mu l_{j} * \eta^{\varepsilon}\right)(y)}{\left(\mu l_{j} * \eta^{\varepsilon}\right)(y)}$ and $\frac{\mathrm{d} \nu}{\mathrm{d} \mathcal{L}^{d}}(x, y)=\eta^{\varepsilon}(y-x) \mu \iota_{j}(x)$, we get for $p \geq 2$

$$
\begin{aligned}
& \iint\left|\frac{\nabla \mu \iota_{j}(x)}{\mu\left\llcorner_{j}(x)\right.}-\frac{\nabla\left(\mu\left\llcorner_{j} * \eta^{\varepsilon}\right)(y)\right.}{\left(\mu\left\llcorner_{j} * \eta^{\varepsilon}\right)(y)\right.}\right|^{p} \eta^{\varepsilon}(y-x) \mu \iota_{j}(x) \mathrm{d} x \mathrm{~d} y \\
& \leq 2^{p-1} \iint\left|\frac{\nabla \mu \downarrow_{j}(x)}{\mu\left\llcorner_{j}(x)\right.}\right|^{p} \eta^{\varepsilon}(y-x) \mu \iota_{j}(x) \mathrm{d} x \mathrm{~d} y \\
& +\left.2^{p-1} \iint\left|\frac{\nabla\left(\mu \iota_{j} * \eta^{\varepsilon}\right)(y)}{\left(\mu \iota_{j} * \eta^{\varepsilon}\right)(y)}\right|^{p} \eta^{\varepsilon}(y-x) \mu\right|_{j}(x) \mathrm{d} x \mathrm{~d} y \\
& -\iint\left|\frac{\nabla \mu\left\llcorner_{j}(x)\right.}{\mu\left\llcorner_{j}(x)\right.}+\frac{\nabla\left(\mu\left\llcorner_{j} * \eta^{\varepsilon}\right)(y)\right.}{\left(\mu\left\llcorner_{j} * \eta^{\varepsilon}\right)(y)\right.}\right|^{p} \eta^{\varepsilon}(y-x) \mu\left\llcorner_{j}(x) \mathrm{d} x \mathrm{~d} y\right. \\
& =2^{p-1} \int \frac{\left|\nabla \mu \iota_{j}(x)\right|^{p}}{\mu \iota_{j}(x)^{p-1}} \mathrm{~d} x+2^{p-1} \int \frac{\left|\nabla\left(\left.\mu\right|_{j} * \eta^{\varepsilon}\right)(y)\right|^{p}}{\left(\mu \iota_{j} * \eta^{\varepsilon}\right)(y)^{p-1}} \mathrm{~d} y \\
& -\iint\left|\frac{\nabla \mu \iota_{j}(x)}{\mu \iota_{j}(x)}+\frac{\nabla\left(\mu \iota_{j} * \eta^{\varepsilon}\right)(y)}{\left(\mu \iota_{j} * \eta^{\varepsilon}\right)(y)}\right|^{p} \eta^{\varepsilon}(y-x) \mu \iota_{j}(x) \mathrm{d} x \mathrm{~d} y .
\end{aligned}
$$

On the other hand, using (6.9), for $1<p<2$ we have

$$
\begin{aligned}
& \iint\left|\frac{\nabla \mu\left\llcorner_{j}(x)\right.}{\mu \iota_{j}(x)}-\frac{\nabla\left(\mu\left\llcorner_{j} * \eta^{\varepsilon}\right)(y)\right.}{\left(\mu\left\llcorner_{j} * \eta^{\varepsilon}\right)(y)\right.}\right|^{p} \eta^{\varepsilon}(y-x) \mu\left\llcorner_{j}(x) \mathrm{d} x \mathrm{~d} y\right. \\
& \leq\left[\left(2^{p-1} \int \frac{\left|\nabla \mu \downarrow_{j}(x)\right|^{p}}{\mu \downarrow_{j}(x)^{p-1}} \mathrm{~d} x+2^{p-1} \int \frac{\mid \nabla\left(\left.\mu\left\llcorner_{j} * \eta^{\varepsilon}\right)(y)\right|^{p}\right.}{\left(\mu \downarrow_{j} * \eta^{\varepsilon}\right)(y)^{p-1}} \mathrm{~d} y\right)^{\frac{1}{p-1}}\right. \\
& \left.-\left(\iint\left|\frac{\nabla \mu \iota_{j}(x)}{\mu \iota_{j}(x)}+\frac{\nabla\left(\mu \downarrow_{j} * \eta^{\varepsilon}\right)(y)}{\left(\mu\left\llcorner_{j} * \eta^{\varepsilon}\right)(y)\right.}\right|^{p} \eta^{\varepsilon}(y-x) \mu \iota_{j}(x) \mathrm{d} x \mathrm{~d} y\right)^{\frac{1}{p-1}}\right]^{p-1} \text {. }
\end{aligned}
$$

Finally, by convexity of the function $z \mapsto|z|^{p}$ on $\mathbb{R}^{d}$ we have

$$
\begin{aligned}
& \iint\left|\frac{\nabla \mu \iota_{j}(x)}{\mu \iota_{j}(x)}+\frac{\nabla\left(\mu \iota_{j} * \eta^{\varepsilon}\right)(y)}{\left(\mu \iota_{j} * \eta^{\varepsilon}\right)(y)}\right|^{p} \eta^{\varepsilon}(y-x) \mu \iota_{j}(x) \mathrm{d} x \mathrm{~d} y \\
& \geq \iint\left|\frac{2 \nabla\left(\mu \mathrm{l}_{j} * \eta^{\varepsilon}\right)(y)}{\left(\mu \mathrm{l}_{j} * \eta^{\varepsilon}\right)(y)}\right|^{p} \eta^{\varepsilon}(y-x) \mu \mathrm{l}_{j}(x) \mathrm{d} x \mathrm{~d} y \\
& +2^{p-1} p \sum_{j=1}^{d} \iint\left|\frac{\partial_{j}\left(\mu \iota_{j} * \eta^{\varepsilon}\right)(y)}{\left(\mu \iota_{j} * \eta^{\varepsilon}\right)(y)}\right|^{p-2} \frac{\partial_{j}\left(\mu \iota_{j} * \eta^{\varepsilon}\right)(y)}{\left(\mu\left\llcorner_{j} * \eta^{\varepsilon}\right)(y)\right.} \frac{\partial_{j} \mu \iota_{j}(x)}{\mu \iota_{j}(x)} \eta^{\varepsilon}(y-x) \mu \iota_{j}(x) \mathrm{d} x \mathrm{~d} y \\
& -2^{p-1} p \sum_{j=1}^{d} \iint\left|\frac{\partial_{j}\left(\mu\left\llcorner_{j} * \eta^{\varepsilon}\right)(y)\right.}{\left(\mu\left\llcorner_{j} * \eta^{\varepsilon}\right)(y)\right.}\right|^{p-2} \frac{\left|\partial_{j}\left(\left.\mu\right|_{j} * \eta^{\varepsilon}\right)(y)\right|^{2}}{\left(\mu\left\llcorner_{j} * \eta^{\varepsilon}\right)(y)^{2}\right.} \eta^{\varepsilon}(y-x) \mu\left\llcorner_{j}(x) \mathrm{d} x \mathrm{~d} y\right. \\
& =2^{p} \int \frac{\left|\nabla\left(\mu \iota_{j} * \eta^{\varepsilon}\right)(y)\right|^{p}}{\left(\mu \iota_{j} * \eta^{\varepsilon}\right)(y)^{p-1}} \mathrm{~d} y \text {. }
\end{aligned}
$$

Hence, for $p \geq 2$,

$$
\begin{aligned}
& \iint\left|\frac{\nabla \mu\left\llcorner_{j}(x)\right.}{\mu \iota_{j}(x)}-\frac{\nabla\left(\mu\left\llcorner_{j} * \eta^{\varepsilon}\right)(y)\right.}{\left(\mu\left\llcorner_{j} * \eta^{\varepsilon}\right)(y)\right.}\right|^{p} \eta^{\varepsilon}(y-x) \mu \iota_{j}(x) \mathrm{d} x \mathrm{~d} y \\
& \leq 2^{p-1}\left(\int \frac{\left.|\nabla \mu|_{j}(x)\right|^{p}}{\mu \iota_{j}(x)^{p-1}} \mathrm{~d} x-\int \frac{\left|\nabla\left(\left.\mu\right|_{j} * \eta^{\varepsilon}\right)(y)\right|^{p}}{\left(\left.\mu\right|_{j} * \eta^{\varepsilon}\right)(y)^{p-1}} \mathrm{~d} y\right) \\
& =2^{p-1} p^{p}\left(\mathcal{E}^{1, p}\left(\left.\mu\right|_{j}\right)-\mathcal{E}^{1, p}\left(\left.\mu\right|_{j} * \eta^{\varepsilon}\right)\right) \text {, }
\end{aligned}
$$


while for $1<p<2$

$$
\begin{aligned}
& \iint\left|\frac{\nabla \mu\left\llcorner_{j}(x)\right.}{\mu \iota_{j}(x)}-\frac{\nabla\left(\mu\left\llcorner_{j} * \eta^{\varepsilon}\right)(y)\right.}{\left(\mu \iota_{j} * \eta^{\varepsilon}\right)(y)}\right|^{p} \eta^{\varepsilon}(y-x) \mu \iota_{j}(x) \mathrm{d} x \mathrm{~d} y \\
& \leq 2^{p-1} p^{p}\left[\left(\mathcal{E}^{1, p}\left(\mu\left\llcorner_{j}\right)+\mathcal{E}^{1, p}\left(\mu \iota_{j} * \eta^{\varepsilon}\right)\right)^{\frac{1}{p-1}}-2 \mathcal{E}^{1, p}\left(\mu \iota_{j} * \eta^{\varepsilon}\right)^{\frac{1}{p-1}}\right]^{p-1},\right.
\end{aligned}
$$

Putting all together and summing on $j$ we get the thesis with $c_{p}=2^{\frac{p-1}{p}}$.

Remark 3. As one would expect, if the measure $\mu$ is not regular then the bound on the energy of $\Theta^{\varepsilon}[\mu]$ diverges as $\varepsilon$ approaches zero, as in (6.3) and (6.6). On the contrary, if $\mu$ is $W^{1, p}$-regular then the bound on the energy of $\Theta^{\varepsilon}[\mu]$ in (6.4) and (6.7) converges to the energy of $\mu$ as $\varepsilon \rightarrow 0$. Indeed, on the one hand $\Delta(\varepsilon, p, \mu)$ converges to zero by Proposition 4.2 On the other hand, let $\lambda^{\varepsilon}\left(z_{1}, \ldots, z_{N}\right)=$ $\eta^{\varepsilon}\left(z_{1}\right) \cdots \eta^{\varepsilon}\left(z_{N}\right)$, we have $\Lambda^{\varepsilon}[\mu]=\mu * \lambda^{\varepsilon}$, and hence

$$
\left\|\nabla_{x_{j}}\left(\mu * \lambda^{\varepsilon}\right)^{\frac{1}{p}}\right\|_{p} \rightarrow\left\|\nabla_{x_{j}} \mu^{\frac{1}{p}}\right\|_{p} .
$$

When we raise to the power $p$ and sum over $j$ we get $\mathcal{E}^{1, p}(\mu)$ in view of the usual condition (2.3).

\section{Continuity of $\Theta$ IN $\varepsilon$}

Finally, in this section we prove that $\Theta$ satisfies property $\mathrm{C}$ of Section 5 , In order to simplify the notation, let as above $P^{\varepsilon}[\mu]$ be the measure over $\left(\mathbb{R}^{d}\right)^{N} \times\left(\mathbb{R}^{d}\right)^{N}$ given by

$$
\iint \psi(X, Y) \mathrm{d} P^{\varepsilon}[\mu](X, Y):=\left.\iint \psi(X, Y) \prod_{k=1}^{N} \frac{\eta^{\varepsilon}\left(y_{k}-x_{k}\right)}{\left(\mu L_{k} * \eta^{\varepsilon}\right)\left(y_{k}\right)} \mathrm{d} \mu\right|_{k}\left(x_{k}\right) \mathrm{d} \Lambda^{\varepsilon}[\mu](Y),
$$

already introduced in Section 6] and let $Q^{\varepsilon}[\mu]$ be the measure over $\left(\mathbb{R}^{d}\right)^{N} \times\left(\mathbb{R}^{d}\right)^{N}$ given by

$$
\iint \psi(X, Y) \mathrm{d} Q^{\varepsilon}[\mu](X, Y):=\iint \psi(X, Y) \prod_{k=1}^{N} \eta^{\varepsilon}\left(y_{k}-x_{k}\right) \mathrm{d} \mu(X) \mathrm{d} Y
$$

for any $\psi:\left(\mathbb{R}^{d}\right)^{N} \times\left(\mathbb{R}^{d}\right)^{N} \rightarrow \mathbb{R}$ bounded and countinuous.

Remark 4. Notice that, if $\psi \in C_{b}\left(\left(\mathbb{R}^{d}\right)^{N}\right)$, then recalling definitions (5.1) and (5.2) we have

$$
\begin{aligned}
\iint \psi(X) \mathrm{d} P^{\varepsilon}[\mu](X, Y) & =\iint \psi(X) \prod_{k=1}^{N} \frac{\eta^{\varepsilon}\left(y_{k}-x_{k}\right)}{\left(\mu \iota_{k} * \eta^{\varepsilon}\right)\left(y_{k}\right)} \mathrm{d} \mu \iota_{k}\left(x_{k}\right) \mathrm{d} \Lambda^{\varepsilon}[\mu](Y) \\
& =\int \psi(X) \mathrm{d} \Theta^{\varepsilon}[\mu](X),
\end{aligned}
$$

while

$$
\begin{aligned}
\iint \psi(Y) \mathrm{d} P^{\varepsilon}[\mu](X, Y) & =\left.\iint \psi(Y) \prod_{k=1}^{N} \frac{\eta^{\varepsilon}\left(y_{k}-x_{k}\right)}{\left(\left.\mu\right|_{k} * \eta^{\varepsilon}\right)\left(y_{k}\right)} \mathrm{d} \mu\right|_{k}\left(x_{k}\right) \mathrm{d} \Lambda^{\varepsilon}[\mu](Y) \\
& =\int \psi(Y) \mathrm{d} \Lambda^{\varepsilon}[\mu](Y) .
\end{aligned}
$$

On the other hand,

$$
\begin{aligned}
\iint \psi(X) \mathrm{d} Q^{\varepsilon}[\mu](X, Y) & =\iint \psi(X) \prod_{k=1}^{N} \eta^{\varepsilon}\left(y_{k}-x_{k}\right) \mathrm{d} \mu(X) \mathrm{d} Y \\
& =\int \psi(X) \mathrm{d} \mu(X)
\end{aligned}
$$


while

$$
\begin{aligned}
\iint \psi(Y) \mathrm{d} Q^{\varepsilon}[\mu](X, Y) & =\iint \psi(Y) \prod_{k=1}^{N} \eta^{\varepsilon}\left(y_{k}-x_{k}\right) \mathrm{d} \mu(X) \mathrm{d} Y \\
& =\int \psi(Y) \mathrm{d} \Lambda^{\varepsilon}[\mu](Y) .
\end{aligned}
$$

Let us introduce a couple of technical results.

Lemma 7.1. There exists a constant $K(d)$, depending only on the dimension $d$, such that for every $\varepsilon, \tau>0$,

$$
\int_{\{|z| \geq \tau\}} \eta^{\varepsilon}(z) \mathrm{d} z \leq K(d) e^{-\frac{\tau^{2}}{4 \varepsilon}}
$$

Proof. It is just a computation: passing to spherical coordinates and denoting by $\sigma_{d}$ the surface area of the unit sphere in $\mathbb{R}^{d}$,

$$
\begin{aligned}
\int_{\{|z| \geq \tau\}} \eta^{\varepsilon}(z) \mathrm{d} z & =\frac{\sigma_{d}}{(2 \pi \varepsilon)^{\frac{d}{2}}} \int_{\tau}^{+\infty} r^{d-1} e^{-\frac{r^{2}}{2 \varepsilon}} \mathrm{d} r \\
& =\frac{\sigma_{d}}{2 \pi^{\frac{d}{2}}} \int_{\frac{\tau^{2}}{2 \varepsilon}}^{+\infty} s^{\frac{d-2}{2}} e^{-s} \mathrm{~d} s \\
& \leq \frac{\sigma_{d}}{2 \pi^{\frac{d}{2}}} e^{-\frac{\tau^{2}}{4 \varepsilon}} \int_{\frac{\tau^{2}}{2 \varepsilon}}^{+\infty} s^{\frac{d-2}{2}} e^{-\frac{s}{2}} \mathrm{~d} s \\
& \leq \frac{\sigma_{d}}{2 \pi^{\frac{d}{2}}} e^{-\frac{\tau^{2}}{4 \varepsilon}} \int_{0}^{+\infty} s^{\frac{d-2}{2}} e^{-\frac{s}{2}} \mathrm{~d} s \\
& =\frac{\sigma_{d}}{2}\left(\frac{2}{\pi}\right)^{\frac{d}{2}} \Gamma\left(\frac{d}{2}\right) e^{-\frac{\tau^{2}}{4 \varepsilon}}
\end{aligned}
$$

Lemma 7.2. For every $r, \varepsilon>0$ and for every $\mu \in \mathcal{P}\left(\left(\mathbb{R}^{d}\right)^{N}\right)$,

$$
\begin{aligned}
& P^{\varepsilon}[\mu](\{|X-Y| \geq r\}) \leq N K(d) \exp \left(-\frac{r^{2}}{4 N \varepsilon}\right) \\
& Q^{\varepsilon}[\mu](\{|X-Y| \geq r\}) \leq N K(d) \exp \left(-\frac{r^{2}}{4 N \varepsilon}\right)
\end{aligned}
$$

where $K(d)$ is the constant in Lemma 7.1.

Proof. Observe that

$$
\begin{aligned}
& \left\{(X, Y) \in\left(\mathbb{R}^{d}\right)^{N} \times\left(\mathbb{R}^{d}\right)^{N}|| X-Y \mid \geq r\right\} \\
& \subseteq \bigcup_{j=1}^{N}\left\{(X, Y) \in\left(\mathbb{R}^{d}\right)^{N} \times\left(\mathbb{R}^{d}\right)^{N}|| x_{j}-y_{j} \mid \geq \frac{r}{\sqrt{N}}\right\}
\end{aligned}
$$


Using Lemma 7.1, this yields

$$
\begin{aligned}
P^{\varepsilon}[\mu](\{|X-Y| \geq r\}) & \leq \sum_{j=1}^{N} P^{\varepsilon}[\mu]\left(\left\{\left|x_{j}-y_{j}\right| \geq \frac{r}{\sqrt{N}}\right\}\right) \\
& =\left.\sum_{j=1}^{N} \int_{\left\{\left|x_{j}-y_{j}\right| \geq \frac{r}{\sqrt{N}}\right\}} \prod_{k=1}^{N} \frac{\eta^{\varepsilon}\left(y_{k}-x_{k}\right)}{\left(\left.\mu\right|_{k} * \eta^{\varepsilon}\right)\left(y_{k}\right)} \mathrm{d} \mu\right|_{k}\left(x_{k}\right) \mathrm{d} \Lambda^{\varepsilon}[\mu](Y) \\
& =\left.\sum_{j=1}^{N} \int_{\left\{\left|x_{j}-y_{j}\right| \geq \frac{r}{\sqrt{N}}\right\}} \eta^{\varepsilon}\left(y_{j}-x_{j}\right) \mathrm{d} \mu\right|_{j}\left(x_{j}\right) \mathrm{d} y_{j} \\
& =\left.\sum_{j=1}^{N} \int_{\left\{\left|z_{j}\right| \geq \frac{r}{\sqrt{N}}\right\}} \eta^{\varepsilon}\left(z_{j}\right) \mathrm{d} \mu\right|_{j}\left(x_{j}\right) \mathrm{d} z_{j} \\
& =N \int_{\left\{|z| \geq \frac{r}{\sqrt{N}}\right\}} \eta^{\varepsilon}(z) \mathrm{d} z \leq N K(d) \exp \left(-\frac{r^{2}}{4 N \varepsilon}\right) .
\end{aligned}
$$

Analogously,

$$
\begin{aligned}
Q^{\varepsilon}[\mu](\{|X-Y| \geq r\}) & \leq \sum_{j=1}^{N} Q^{\varepsilon}[\mu]\left(\left\{\left|x_{j}-y_{j}\right| \geq \frac{r}{\sqrt{N}}\right\}\right) \\
& =\sum_{j=1}^{N} \int_{\left\{\left|x_{j}-y_{j}\right| \geq \frac{r}{\sqrt{N}}\right\}} \prod_{k=1}^{N} \eta^{\varepsilon}\left(y_{k}-x_{k}\right) \mathrm{d} \mu(X) \mathrm{d} Y \\
& =\left.\sum_{j=1}^{N} \int_{\left\{\left|x_{j}-y_{j}\right| \geq \frac{r}{\sqrt{N}}\right\}} \eta^{\varepsilon}\left(y_{j}-x_{j}\right) \mathrm{d} \mu\right|_{j}\left(x_{j}\right) \mathrm{d} y_{j} \\
& =\sum_{j=1}^{N} \int_{\left\{\left|z_{j}\right| \geq \frac{r}{\sqrt{N}}\right\}} \eta^{\varepsilon}\left(z_{j}\right) \mathrm{d} \mu L_{j}\left(x_{j}\right) \mathrm{d} z_{j} \\
& =N \int_{\left\{|z| \geq \frac{r}{\sqrt{N}}\right\}} \eta^{\varepsilon}(z) \mathrm{d} z \leq N K(d) \exp \left(-\frac{r^{2}}{4 N \varepsilon}\right) .
\end{aligned}
$$

We now move towards the proof of property C. Even though it requires to test the convergence of $\Theta^{\varepsilon}[\mu]$ to $\mu$ for all the continuous and bounded functions, first we prove the convergence for a smaller class, namely the continuous functions with compact support.

Proposition 7.3. Let $\mu \in \mathcal{P}\left(\left(\mathbb{R}^{d}\right)^{N}\right)$. Then, for every $\psi \in C_{c}\left(\left(\mathbb{R}^{d}\right)^{N}\right)$,

$$
\lim _{\varepsilon \rightarrow 0} \int \psi(X) \mathrm{d} \Theta^{\varepsilon}[\mu](X)=\int \psi(X) \mathrm{d} \mu(X) .
$$

Proof. Fix $\psi:\left(\mathbb{R}^{d}\right)^{N} \rightarrow \mathbb{R}$ a continuous function with compact support and $\delta>0$. Since $\psi$ is absolutely continuous, let $\varepsilon_{0}>0$ be such that

$$
|X-Y|<\varepsilon_{0}^{\frac{1}{4}} \Longrightarrow|\psi(X)-\psi(Y)|<\delta
$$


Using Remark 4 we have:

$$
\begin{aligned}
& \left|\int \psi(X) \mathrm{d} \Theta^{\varepsilon}[\mu](X)-\int \psi(X) \mathrm{d} \mu(X)\right| \\
& \leq\left|\int \psi(X) \mathrm{d} \Theta^{\varepsilon}[\mu](X)-\int \psi(Y) \mathrm{d} \Lambda^{\varepsilon}[\mu](Y)\right| \\
& \quad+\left|\int \psi(Y) \mathrm{d} \Lambda^{\varepsilon}[\mu](Y)-\int \psi(X) \mathrm{d} \mu(X)\right| \\
& \leq \iint|\psi(X)-\psi(Y)| \mathrm{d} P^{\varepsilon}[\mu](X, Y)+\iint|\psi(Y)-\psi(X)| \mathrm{d} Q^{\varepsilon}[\mu](X, Y) .
\end{aligned}
$$

Let us put

$$
\begin{aligned}
& A^{\varepsilon}=\left\{(X, Y) \in\left(\mathbb{R}^{d}\right)^{N} \times\left(\mathbb{R}^{d}\right)^{N}:|X-Y| \geq \varepsilon^{\frac{1}{4}}\right\} \\
& B^{\varepsilon}=\left\{(X, Y) \in\left(\mathbb{R}^{d}\right)^{N} \times\left(\mathbb{R}^{d}\right)^{N}:|X-Y|<\varepsilon^{\frac{1}{4}}\right\} .
\end{aligned}
$$

Using Lemma 7.2,

$$
\begin{aligned}
\iint_{A^{\varepsilon}}|\psi(X)-\psi(Y)| \mathrm{d} P^{\varepsilon}[\mu](X, Y) & \leq 2\|\psi\|_{\infty} P^{\varepsilon}[\mu]\left(A^{\varepsilon}\right) \\
& \leq 2 N K(d)\|\psi\|_{\infty} \exp \left(-\frac{1}{4 N \sqrt{\varepsilon}}\right) .
\end{aligned}
$$

which goes to zero as $\varepsilon \rightarrow 0$. On the other hand, for every $\varepsilon<\varepsilon_{0}$ we have

$$
\iint_{B^{\varepsilon}}|\psi(X)-\psi(Y)| \mathrm{d} P^{\varepsilon}[\mu](X, Y) \leq \delta P^{\varepsilon}[\mu]\left(B^{\varepsilon}\right) \leq \delta .
$$

Treating the integral with respect to the measure $Q^{\varepsilon}[\mu]$ in the same way we get the thesis since $\delta$ was arbitrary.

One way to extend the result of Proposition 7.3 to the continuous and bounded functions is to use the Prokhorov's theorem (Theorem 2.1), by first proving that, for every $\mu \in \mathcal{P}\left(\left(\mathbb{R}^{d}\right)^{N}\right)$, the family $\left\{\Theta^{\varepsilon}(\mu)\right\}_{\varepsilon>0}$ is tight. In view of Lemma 5.2, this is actually a simple corollary of the following more general result.

Theorem 7.4. Let $\mathcal{M} \subseteq \mathcal{P}\left(\left(\mathbb{R}^{d}\right)^{N}\right)$ such that, for every $\mu, \nu \in \mathcal{M}$ and every $j=1, \ldots, N$,

\section{Then $\mathcal{M}$ is tight.}

$$
\left.\mu\right|_{j}=\left.\nu\right|_{j}
$$

Proof. Let $\rho_{1}, \ldots, \rho_{N}$ be the common marginals of all the measures in $\mathcal{M}$, and fix $\delta>0$. Since every $\rho_{j}$ is a probability, we may find $K \subseteq \mathbb{R}^{d}$ compact such that $\rho_{j}(K) \geq 1-\frac{\delta}{N}$ for all $j=1, \ldots, N$. Let $K^{N}:=K \times \cdots \times K \subseteq\left(\mathbb{R}^{d}\right)^{N}$, which is compact. We claim that $\mu\left(K^{N}\right) \geq 1-\delta$ for all $\mu \in \mathcal{M}$. First notice that

$$
\left(K^{N}\right)^{c}=\bigcup_{j=1}^{N}\left(\mathbb{R}^{d} \times \cdots \times \underset{\substack{\uparrow \\ j \text {-th }}}{K^{c}} \times \cdots \times \mathbb{R}^{d}\right) .
$$

Hence, for every $\mu \in \mathcal{M}$,

$$
\begin{aligned}
& \mu\left(\left(K^{N}\right)^{c}\right) \leq \sum_{j=1}^{N} \mu\left(\mathbb{R}^{d} \times \cdots \times \underset{\substack{\uparrow \\
j \text {-th }}}{K^{c}} \times \cdots \times \mathbb{R}^{d}\right) \\
& =\sum_{j=1}^{N} \mu \iota_{j}\left(K^{c}\right)=\sum_{j=1}^{N} \rho_{j}\left(K^{c}\right) \leq \sum_{j=1}^{N} \frac{\delta}{N}=\delta,
\end{aligned}
$$

so that $\mu\left(K^{N}\right) \geq 1-\delta$. 
Finally combining Theorem 2.1 with Proposition 7.3 we get the convergence of $\Theta^{\varepsilon}[\mu]$ to $\mu$ in duality with $C_{b}\left(\left(\mathbb{R}^{d}\right)^{N}\right)$, as wanted.

Theorem 7.5. Let $\mu \in \mathcal{P}\left(\left(\mathbb{R}^{d}\right)^{N}\right)$. Then, for every $\psi \in C_{b}\left(\left(\mathbb{R}^{d}\right)^{N}\right)$,

$$
\lim _{\varepsilon \rightarrow 0} \int \psi(X) \mathrm{d} \Theta^{\varepsilon}[\mu](X)=\int \psi(X) \mathrm{d} \mu(X) .
$$

Proof. Suppose by contradiction that there exists $\delta>0$, a sequence $\varepsilon_{n} \searrow 0$ and a continuous bounded function $\psi:\left(\mathbb{R}^{d}\right)^{N} \rightarrow \mathbb{R}$ such that

$$
\left|\int \psi(X) \mathrm{d} \Theta^{\varepsilon_{n}}[\mu](X)-\int \psi(X) \mathrm{d} \mu(X)\right| \geq \delta>0 .
$$

Denote for simplicity $\mu_{n}:=\Theta^{\varepsilon_{n}}[\mu]$. We know that the family $\left\{\mu_{n}\right\}_{n \in \mathbb{N}}$ is tight, and by Theorem 2.1 we may extract a subsequence $\mu_{n_{k}}$ weakly converging to some $\nu \in \mathcal{P}\left(\left(\mathbb{R}^{d}\right)^{N}\right)$. However Proposition 7.3 ensures that $\nu=\mu$, and hence $\mu_{n_{k}} \rightarrow \mu$, contradicting (7.1).

We conclude this section with a final result about the continuity of $\Theta$. We proved in Theorem 7.5 that $\Theta^{\varepsilon}[\mu] \rightarrow \mu$ as $\varepsilon \rightarrow 0$, which is the natural notion of convergence as far as $\mu$ is no more regular than a measure. However if $\mu$ has some better regurality, say $\mu \in \mathcal{P}^{1, p}\left(\left(\mathbb{R}^{d}\right)^{N}\right)$, since $\Theta^{\varepsilon}[\mu] \in \mathcal{P}^{1, p}$ for every $\varepsilon>0$ it is natural to ask whether $\Theta^{\varepsilon}[\mu] \rightarrow \mu$ in the $d^{1, p}$-topology. The answer is positive, as stated in the following

Theorem 7.6. Let $\mu \in \mathcal{P}^{1, p}\left(\left(\mathbb{R}^{d}\right)^{N}\right)$, with $p>1$. Then

$$
\lim _{\varepsilon \rightarrow 0} d^{1, p}\left(\Theta^{\varepsilon}[\mu], \mu\right)=0 \text {. }
$$

Proof. Combining the fact that the family $\Theta^{\varepsilon}[\mu]^{\frac{1}{p}}$ is bounded in $W^{1, p}$ due to Theorem 6.4 and the result of Theorem 7.5 we get that $\Theta^{\varepsilon}[\mu]^{\frac{1}{p}} \rightarrow \mu^{\frac{1}{p}}$ weakly in $W^{1, p}\left(\left(\mathbb{R}^{d}\right)^{N}\right)$ as $\varepsilon \rightarrow 0$. Since $W^{1, p}$ is uniformly convex, we need only to check that

$$
\lim _{\varepsilon \rightarrow 0}\left\|\Theta^{\varepsilon}[\mu]^{\frac{1}{p}}\right\|_{W^{1, p}}=\left\|\mu^{\frac{1}{p}}\right\|_{W^{1, p}} .
$$

The $L^{p}$-norms are identically equal to 1 , so we need to prove the limit for the norms of the gradients. The weak convergence of $\nabla \Theta^{\varepsilon}[\mu]^{\frac{1}{p}}$ to $\nabla \mu^{\frac{1}{p}}$ implies that

$$
\liminf _{\varepsilon \rightarrow 0}\left\|\nabla \Theta^{\varepsilon}[\mu]^{\frac{1}{p}}\right\|_{L^{p}} \geq\left\|\nabla \mu^{\frac{1}{p}}\right\|_{L^{p}} .
$$

The other inequality follows from Remark 3

\section{Continuity of $\Theta$ In $\mu$}

We devote this final section to the proof of Theorem 5.1. Throughout this section, $\varepsilon$ will be fixed and positive. The main idea for the proof of Theorem 5.1 is to use Lebesgue's dominated convergence theorem, but in order to do so we must have some fine upper-bound on the integral kernel $P^{\varepsilon}[\mu]$ defining $\Theta^{\varepsilon}[\mu]$. We refer to (5.1) and (5.2) for the definitions. With a slight abuse of notation, since $\Lambda^{\varepsilon}[\mu]$ and $\left.\mu\right|_{j} * \eta^{\varepsilon}$ are absolutely continuous with respect to the Lebesgue measure, we will use the same symbol for the measure and its density.

Lemma 8.1. Let $\mu \in \mathcal{P}\left(\left(\mathbb{R}^{d}\right)^{N}\right)$. Then:

$$
\Lambda^{\varepsilon}[\mu](Y) \leq(2 \pi \varepsilon)^{-\frac{(N-1) d}{2 N}} \prod_{k=1}^{N}\left(\mu \downarrow_{k} * \eta^{\varepsilon}\right)\left(y_{k}\right)^{\frac{1}{N}} .
$$


(ii) Let $R>0, \gamma \in[0,1]$ be such that $\mu\left\llcorner_{j}(B(0, R)) \geq \gamma\right.$. Then

$$
\left(\mu l_{j} * \eta^{\varepsilon}\right)\left(y_{j}\right) \geq \frac{\gamma}{(2 \pi \varepsilon)^{d / 2}} \exp \left(-\frac{\left(\left|y_{j}\right|+R\right)^{2}}{2 \varepsilon}\right) .
$$

Proof. (i) We apply a general version of the Hölder's inequality with exponents $p_{1}=\cdots p_{N}=N$, and use the fact that $\eta^{\varepsilon}(z) \leq \eta^{\varepsilon}(0)=(2 \pi \varepsilon)^{-d / 2}$, to get

$$
\begin{aligned}
\Lambda^{\varepsilon}[\mu](Y) & =\int \prod_{j=1}^{N} \eta^{\varepsilon}\left(y_{j}-x_{j}\right) \mathrm{d} \mu(X) \\
& \leq \prod_{j=1}^{N}\left(\int \eta^{\varepsilon}\left(y_{j}-x_{j}\right)^{N} \mathrm{~d} \mu(X)\right)^{\frac{1}{N}} \\
& \leq(2 \pi \varepsilon)^{-\frac{(N-1) d}{2 N}} \prod_{j=1}^{N}\left(\int \eta^{\varepsilon}\left(y_{j}-x_{j}\right) \mathrm{d} \mu(X)\right)^{\frac{1}{N}} \\
& =(2 \pi \varepsilon)^{-\frac{(N-1) d}{2 N}} \prod_{j=1}^{N}\left(\mu L_{j} * \eta^{\varepsilon}\right)\left(y_{j}\right)^{\frac{1}{N}} .
\end{aligned}
$$

as wanted.

(ii) We start observing that

$$
\left(\mu \iota_{j} * \eta^{\varepsilon}\right)\left(y_{j}\right)=\int \eta^{\varepsilon}\left(y_{j}-x_{j}\right) \mathrm{d} \mu \iota_{j}\left(x_{j}\right) \geq \int_{B(0, R)} \eta^{\varepsilon}\left(y_{j}-x_{j}\right) \mathrm{d} \mu \iota_{j}\left(x_{j}\right) .
$$

When $x_{j}$ belongs to the ball $B(0, R)$, the minimum value of $\eta^{\varepsilon}\left(y_{j}-x_{j}\right)$ is attained at $x_{j}=-R \frac{y_{j}}{\left|y_{j}\right|}$, or at any boundary point if $y_{j}=0$. Thus, in this region,

$$
\eta^{\varepsilon}\left(y_{j}-x_{j}\right) \geq \frac{1}{(2 \pi \varepsilon)^{d / 2}} \exp \left(-\frac{\left(\left|y_{j}\right|+R\right)^{2}}{2 \varepsilon}\right)
$$

and the thesis follows easily.

Lemma 8.2. Let $\rho_{n}, \rho \in \mathcal{P}^{1, p}\left(\mathbb{R}^{d}\right)$ such that $\rho_{n} \rightarrow \rho$ in the $d^{1, p}$-topology. Then the family $\{\rho\} \cup\left\{\rho_{n}\right\}_{n \in \mathbb{N}}$ is tight. In particular, for every $\gamma>0$ there exists $R>0$ such that $\rho_{n}(B(0, R)) \geq 1-\gamma$ and $\rho(B(0, R)) \geq 1-\gamma$.

Proof. Due to Prokhorov's theorem (Theorem 2.1), it suffices to show that $\rho_{n} \rightarrow \rho$. However, by Lemma 3.3 we have the stronger property $\rho_{n} \rightarrow \rho$ in $W^{1,1}\left(\mathbb{R}^{d}\right)$.

Proposition 8.3. Suppose that $\mu^{n} \rightarrow \mu$, with $\left.\left.\mu^{n}\right|_{j} \rightarrow \mu\right|_{j}$ in $\mathcal{P}^{1, p}\left(\mathbb{R}^{d}\right)$ and $\left.\mu^{n}\right|_{j} \rightarrow$ $\mu \iota_{j}$ pointwise a.e. on $\mathbb{R}^{d}$ for every $j=1, \ldots, N$. Then $\Theta^{\varepsilon}\left[\mu^{n}\right] \rightarrow \Theta^{\varepsilon}[\mu]$ pointwise a.e. on $\left(\mathbb{R}^{d}\right)^{N}$.

Assume in addiction that $\left.\nabla \mu^{n} L_{j} \rightarrow \nabla \mu\right|_{j}$ pointwise a.e. on $\mathbb{R}^{d}$. Then $\nabla \Theta^{\varepsilon}\left[\mu^{n}\right] \rightarrow$ $\nabla \Theta^{\varepsilon}[\mu]$ pointwise a.e. on $\left(\mathbb{R}^{d}\right)^{N}$.

Proof. Let $P^{\varepsilon}[\mu](X, Y)$ be the integral kernel defining $\Theta^{\varepsilon}[\mu]$, namely

$$
P^{\varepsilon}[\mu](X, Y)=\prod_{j=1}^{N} \frac{\eta^{\varepsilon}\left(y_{j}-x_{j}\right)}{\left(\mu \iota_{j} * \eta^{\varepsilon}\right)\left(y_{j}\right)} \mu \iota_{j}\left(x_{j}\right) \Lambda^{\varepsilon}[\mu](Y) .
$$

We claim that $P^{\varepsilon}\left[\mu^{n}\right]$ converges pointwise a.e. to $P^{\varepsilon}[\mu]$. For every $Y \in\left(\mathbb{R}^{d}\right)^{N}$ and every $j \in\{1, \ldots, N\}$ we have

$$
\begin{aligned}
\left|\left(\mu^{n} L_{j} * \eta^{\varepsilon}\right)\left(y_{j}\right)-\left(\mu \iota_{j} * \eta^{\varepsilon}\right)\left(y_{j}\right)\right| & \leq \int \eta^{\varepsilon}\left(y_{j}-x_{j}\right)\left|\mu^{n}\right|_{j}\left(x_{j}\right)-\left.\mu\right|_{j}\left(x_{j}\right) \mid \mathrm{d} x_{j} \\
& \leq \frac{1}{(2 \pi \varepsilon)^{\frac{d}{2}}}\left\|\left.\mu^{n}\right|_{j}-\left.\mu\right|_{j}\right\|_{1} \rightarrow 0
\end{aligned}
$$


by Proposition 2.4. Moreover

$$
\begin{aligned}
& \left|\Lambda^{\varepsilon}\left[\mu^{n}\right](Y)-\Lambda^{\varepsilon}[\mu](Y)\right| \\
& \leq\left|\int \prod_{j=1}^{N} \eta^{\varepsilon}\left(y_{j}-x_{j}\right) \mathrm{d} \mu^{n}(X)-\int \prod_{j=1}^{N} \eta^{\varepsilon}\left(y_{j}-x_{j}\right) \mathrm{d} \mu(X)\right|
\end{aligned}
$$

goes to zero for every $Y$ because $\prod \eta^{\varepsilon}\left(y_{j}-x_{j}\right)$ is a fixed countinuous bounded function, and $\mu^{n} \rightarrow \mu$. Finally fix $X \in\left(\mathbb{R}^{d}\right)^{N}$ in the set of full measure such that $\mu^{n} L_{j}\left(x_{j}\right) \rightarrow \mu L_{j}\left(x_{j}\right)$ for every $j=1, \ldots, N$.

We need only to find a domination for $P^{\varepsilon}\left[\mu^{n}\right]$. For every $j=1, \ldots, N$ let $R_{j}$ given by Lemma 8.2 for $\gamma=\frac{1}{2}$, and let $R=\max _{j} R_{j}$. Using Lemma 8.1 (i) and (ii) one has

$$
\begin{aligned}
P^{\varepsilon}\left[\mu^{n}\right](X, Y) & \leq(2 \pi \varepsilon)^{-\frac{(N-1) d}{2 N}} \prod_{j=1}^{N} \frac{\left.\eta^{\varepsilon}\left(y_{j}-x_{j}\right) \mu^{n}\right|_{j}\left(x_{j}\right)}{\left(\left.\mu^{n}\right|_{j} * \eta^{\varepsilon}\right)\left(y_{j}\right)^{N-1 / N}} \\
& \leq\left. 2^{N} \prod_{j=1}^{N} \eta^{\varepsilon}\left(y_{j}-x_{j}\right) \mu^{n}\right|_{j}\left(x_{j}\right) \exp \left(\frac{(N-1)\left(\left|y_{j}\right|+R\right)^{2}}{2 N \varepsilon}\right) \\
& =\left.2^{N} e^{\frac{(N-1) R^{2}}{2 \varepsilon}} \prod_{j=1}^{N} \mu^{n}\right|_{j}\left(x_{j}\right) e^{\frac{-\left|x_{j}\right|^{2}}{2 \varepsilon}} e^{\frac{-\left|y_{j}\right|^{2}+\left(2 N\left|x_{j}\right|+2(N-1) R\right)\left|y_{j}\right|}{2 N \varepsilon}} .
\end{aligned}
$$

When $X$ and $\varepsilon$ are fixed, the latter is an integrable function of the variable $Y=\left(y_{1}, \ldots, y_{N}\right)$, and we conclude the first part of the proof thanks to Theorem 2.2.

Recalling (6.2) we have

$$
\nabla_{x_{j}} \Theta^{\varepsilon}\left[\mu^{n}\right](X)=\frac{\nabla \mu^{n} L_{j}\left(x_{j}\right)}{\mu^{n} L_{j}\left(x_{j}\right)} \Theta^{\varepsilon}\left[\mu^{n}\right](X)-\int \frac{\nabla \eta^{\varepsilon}\left(y_{j}-x_{j}\right)}{\eta^{\varepsilon}\left(y_{j}-x_{j}\right)} P^{\varepsilon}\left[\mu^{n}\right](X, Y) \mathrm{d} Y
$$

and

$$
\nabla_{x_{j}} \Theta^{\varepsilon}[\mu](X)=\frac{\nabla \mu\left\llcorner_{j}\left(x_{j}\right)\right.}{\mu \iota_{j}\left(x_{j}\right)} \Theta^{\varepsilon}[\mu](X)-\int \frac{\nabla \eta^{\varepsilon}\left(y_{j}-x_{j}\right)}{\eta^{\varepsilon}\left(y_{j}-x_{j}\right)} P^{\varepsilon}[\mu](X, Y) \mathrm{d} Y
$$

Using the first part and the additional assumption on the pointwise convergence of the gradients, we immediately see that

$$
\frac{\left.\nabla \mu^{n}\right|_{j}\left(x_{j}\right)}{\mu^{n} L_{j}\left(x_{j}\right)} \Theta^{\varepsilon}\left[\mu^{n}\right](X) \longrightarrow \frac{\nabla \mu^{n} L_{j}\left(x_{j}\right)}{\mu^{n} L_{j}\left(x_{j}\right)} \Theta^{\varepsilon}\left[\mu^{n}\right](X),
$$

converges pointwise a.e. on $\mathbb{R}^{d} \times \mathbb{R}^{d}$.

As for the second term, like before the integrands converge pointwise a.e., and the domination is obtained using Lemma 8.1 (i) and (ii).

From Proposition 8.3, using some dominations already seen in Section 6, we obtain the following corollary.

Corollary 8.4. Suppose that $\mu^{n} \rightarrow \mu$, with $\left.\left.\mu^{n}\right|_{j} \rightarrow \mu\right|_{j}$ in $\mathcal{P}^{1, p}\left(\mathbb{R}^{d}\right)$ and $\left.\mu^{n}\right|_{j} \rightarrow \mu \iota_{j}$ pointwise a.e. on $\mathbb{R}^{d}$ for every $j=1, \ldots, N$. Then $\left(\Theta^{\varepsilon}\left[\mu^{n}\right]\right)^{\frac{1}{p}} \rightarrow\left(\Theta^{\varepsilon}[\mu]\right)^{\frac{1}{p}}$ in $L^{p}\left(\left(\mathbb{R}^{d}\right)^{N}\right)$.

Assume in addiction that $\left.\left.\nabla \mu^{n}\right|_{j} \rightarrow \nabla \mu\right|_{j}$ pointwise a.e. on $\mathbb{R}^{d}$. Then $\left(\Theta^{\varepsilon}\left[\mu^{n}\right]\right)^{\frac{1}{p}} \rightarrow$ $\left(\Theta^{\varepsilon}[\mu]\right)^{\frac{1}{p}}$ in $W^{1, p}\left(\left(\mathbb{R}^{d}\right)^{N}\right)$.

Proof. By Proposition 8.3 we already have pointwise a.e. convergence of the functions. Using (2.2) we get

$$
\begin{aligned}
\left|\left(\Theta^{\varepsilon}\left[\mu^{n}\right](X)\right)^{\frac{1}{p}}-\left(\Theta^{\varepsilon}[\mu](X)\right)^{\frac{1}{p}}\right|^{p} & \leq\left|\Theta^{\varepsilon}\left[\mu^{n}\right](X)-\Theta^{\varepsilon}[\mu](X)\right| \\
& \leq \Theta^{\varepsilon}\left[\mu^{n}\right](X)+\Theta^{\varepsilon}[\mu](X) .
\end{aligned}
$$


The latter converges pointwise to $2 \Theta^{\varepsilon}[\mu](X)$, and

$$
\int \Theta^{\varepsilon}\left[\mu^{n}\right](X) \mathrm{d} X+\int \Theta^{\varepsilon}[\mu](X) \mathrm{d} X=2,
$$

which allows to conclude the first part of the proof thanks to Theorem 2.2.

Using the expression given by Theorem 6.2 and (2.2) we have

$$
\begin{aligned}
& \int\left|\nabla_{x_{j}}\left(\Theta^{\varepsilon}\left[\mu^{n}\right]\right)^{\frac{1}{p}}(X)-\nabla_{x_{j}}\left(\Theta^{\varepsilon}[\mu]\right)^{\frac{1}{p}}(X)\right|^{p} \mathrm{~d} X \\
& \leq \frac{1}{p^{p}} \int\left|\Theta^{\varepsilon}\left[\mu^{n}\right](X)^{\frac{1-p}{p}} \nabla_{x_{j}} \Theta^{\varepsilon}\left[\mu^{n}\right](X)-\Theta^{\varepsilon}[\mu](X)^{\frac{1-p}{p}} \nabla_{x_{j}} \Theta^{\varepsilon}[\mu](X)\right|^{p} \mathrm{~d} X .
\end{aligned}
$$

By Proposition 8.3 we have pointwise convergence to zero of the integrand. In order to control the gradients we recall Lemma 6.1 and get

$$
\begin{aligned}
\left|\Theta^{\varepsilon}\left[\mu^{n}\right](X)^{\frac{1-p}{p}} \nabla_{x_{j}} \Theta^{\varepsilon}\left[\mu^{n}\right](X)-\Theta^{\varepsilon}[\mu](X)^{\frac{1-p}{p}} \nabla_{x_{j}} \Theta^{\varepsilon}[\mu](X)\right|^{p} \\
\leq 2^{p-1}\left(\Theta^{\varepsilon}\left[\mu^{n}\right](X)^{1-p}\left|\nabla_{x_{j}} \Theta^{\varepsilon}\left[\mu^{n}\right](X)\right|^{p}+\Theta^{\varepsilon}[\mu](X)^{1-p}\left|\nabla_{x_{j}} \Theta^{\varepsilon}[\mu](X)\right|^{p}\right) \\
\leq 4^{p-1}\left(\frac{\left|\nabla \mu^{n} \iota_{j}\left(x_{j}\right)\right|^{p}}{\left.\mu^{n}\right|_{j}\left(x_{j}\right)^{p}} \Theta^{\varepsilon}[\mu](X)+\int \frac{\left|\nabla \eta^{\varepsilon}\left(y_{j}-x_{j}\right)\right|^{p}}{\eta^{\varepsilon}\left(y_{j}-x_{j}\right)^{p}} P^{\varepsilon}\left[\mu^{n}\right](X, Y) \mathrm{d} Y\right) \\
\quad+4^{p-1}\left(\frac{\left|\nabla \mu \iota_{j}\left(x_{j}\right)\right|^{p}}{\mu \iota_{j}\left(x_{j}\right)^{p}} \Theta^{\varepsilon}[\mu](X)+\int \frac{\left|\nabla \eta^{\varepsilon}\left(y_{j}-x_{j}\right)\right|^{p}}{\eta^{\varepsilon}\left(y_{j}-x_{j}\right)^{p}} P^{\varepsilon}[\mu](X, Y) \mathrm{d} Y\right) \\
=: 4^{p-1} g_{n}(X)+4^{p-1} g(X)
\end{aligned}
$$

By hypothesis we have that that $g_{n} \rightarrow g$ pointwise a.e. as in the proof of Proposition 8.3. Moreover, as already seen above,

$$
\int g_{n}(X)=p^{p} \int\left|\nabla\left(\mu^{n} L_{j}\right)^{\frac{1}{p}}\left(x_{j}\right)\right|^{p} \mathrm{~d} x_{j}+\int \frac{\left|\nabla \eta^{\varepsilon}(z)\right|^{p}}{\eta^{\varepsilon}(z)^{p-1}} \mathrm{~d} z
$$

and

$$
\int g(X)=p^{p} \int\left|\nabla\left(\mu \iota_{j}\right)^{\frac{1}{p}}\left(x_{j}\right)\right|^{p} \mathrm{~d} x_{j}+\int \frac{\left|\nabla \eta^{\varepsilon}(z)\right|^{p}}{\eta^{\varepsilon}(z)^{p-1}} \mathrm{~d} z
$$

which allows to conclude thanks to Theorem 2.2

As a final result we obtain Theorem 5.1, which we report here for the sake of the reader.

Theorem 5.1. Let $\mu^{n}, \mu \in \mathcal{P}\left(\left(\mathbb{R}^{d}\right)^{N}\right)$ such that:

(i) $\mu^{n} \rightarrow \mu$ in duality with $C_{b}\left(\left(\mathbb{R}^{d}\right)^{N}\right)$;

(ii) for every $j=1, \ldots, N,\left.\mu^{n}\right|_{j} \in \mathcal{P}^{1, p}\left(\mathbb{R}^{d}\right)$ and $\mu \iota_{j} \in \mathcal{P}^{1, p}\left(\mathbb{R}^{d}\right)$, with

$$
\lim _{n \rightarrow \infty} d^{1, p}\left(\left.\mu^{n}\right|_{j}, \mu L_{j}\right)=0
$$

Then, for every $\varepsilon>0$,

$$
\lim _{n \rightarrow \infty} d^{1, p}\left(\Theta^{\varepsilon}\left[\mu^{n}\right], \Theta^{\varepsilon}[\mu]\right)=0 .
$$

Proof. By contradiction, suppose that there exist $\delta>0$ and a subsequence of $\left(\mu^{n}\right)$ (denoted again $\left(\mu^{n}\right)$ for simplicity) such that

$$
d^{1, p}\left(\Theta^{\varepsilon}\left[\mu^{n}\right], \Theta^{\varepsilon}[\mu]\right) \geq \delta .
$$

Extract a further subsequence $\left(\mu^{n_{k}}\right)_{k}$ such that $\mu^{n_{k}} l_{j} \rightarrow \mu l_{j}$ in $\mathcal{P}^{1, p}\left(\mathbb{R}^{d}\right)$, and in addition $\left.\mu^{n_{k}}\right|_{j} \rightarrow \mu L_{j}$ and $\left.\nabla \mu^{n_{k}}\right|_{j} \rightarrow \nabla \mu L_{j}$ pointwise a.e. on $\mathbb{R}^{d}$ for every $j=1, \ldots, N$. Due to Corollary 8.4 we should have $\left(\Theta^{\varepsilon}\left[\mu^{n_{k}}\right]\right)^{\frac{1}{p}} \rightarrow\left(\Theta^{\varepsilon}[\mu]\right)^{\frac{1}{p}}$ in $W^{1, p}\left(\left(\mathbb{R}^{d}\right)^{N}\right)$, contradicting (8.1). 


\section{REFERENCES}

[1] Luigi Ambrosio and Nicola Gigli. A user's guide to optimal transport. In Modelling and optimisation of flows on networks, pages 1-155. Springer, 2013.

[2] Luigi Ambrosio, Nicola Gigli, and Giuseppe Savaré. Gradient flows: in metric spaces and in the space of probability measures. Springer Science \& Business Media, 2008.

[3] Ugo Bindini. Gamma-convergence and optimal transportation in density functional theory. Master's thesis, Università di Pisa, 2016.

[4] Ugo Bindini and Luigi De Pascale. Optimal transport with coulomb cost and the semiclassical limit of density functional theory. Journal de l'École polytechnique, 4:909-934, 2017.

[5] James A Clarkson. Uniformly convex spaces. Transactions of the American Mathematical Society, 40(3):396-414, 1936.

[6] Codina Cotar, Gero Friesecke, and Claudia Klüppelberg. Density functional theory and optimal transportation with coulomb cost. Communications on Pure and Applied Mathematics, 66(4):548-599, 2013

[7] Codina Cotar, Gero Friesecke, and Claudia Klüppelberg. Smoothing of transport plans with fixed marginals and rigorous semiclassical limit of the hohenberg-kohn functional. Archive for Rational Mechanics and Analysis, pages 1-32, 2018.

[8] Mathieu Lewin. Semi-classical limit of the levy-lieb functional in density functional theory. Comptes Rendus Mathematique, 356(4):449-455, 2018.

[9] Elliott H. Lieb. Density functionals for coulomb systems. In Inequalities, pages 269-303. Springer, 2002

[10] Brendan Pass. Multi-marginal optimal transport: theory and applications. ESAIM: Mathematical Modelling and Numerical Analysis, 49(6):1771-1790, 2015.

Scuola Normale Superiore, Piazza dei Cavalieri, 7, 56126 Pisa - ITALY

E-mail address: ugo.bindini@sns.it 